\title{
Kann die Behandlung mit Quecksilber Cylindrurie und Albuminurie hervorrufen?")
}

Von

Dr. Edvard Welander in Stockholm.

Um ein Heilmittel genau zu kennen, ist es nicht nur nöthig zu wissen, wie es auf einen Theil krankhafte Processe im Organismus einwirken kann, sondern auch - soweit dies möglich jst - auf welchen Wegen es sich in den Körper einführen lässt, wie lange es in ihm remanirt und wann, wie und auf welchen Wegen es eliminirt wird, welche Einwirkung es auf nicht krankhafte veränderte Körpertheile ausübt und welche Ungelegenheiten es in der einen oder anderen Richtung in dem Organismus herbeiführen kann, für den es in Anwendung gebracht werden soll.

In den letzten Jahren sind in dieser Richtung eine Menge Untersuchungen über verschiedene Heilmittel ausgeführt worden; so haben wir eine nicht unbedeutende Kenntniss von der Absorption und Elimination des Quecksilbers erhalten, und ebenso haben wir erfahren, wie dieses Heilmittel - in der einen oder anderen Form angewendet - nachtheilig auf die Haut, auf die Schleimhaut des Mundes, des Magens und des Darmcanals einwirken kann etc. Dahingegen hat man in hohem Grade die Frage übersehen, welche Einwirkung dieses Mittel auf die Nieren haben kann, und dieses ist um so merkwürdiger, da un-

1) Vortrag, gehalten im Verein schwedischer Aerzte am 19. December 1893 . 
zählige Untersuchungen gezeigt haben, dass das Quecksillber constant in grosser Menge gerade durch die Nieren eliminirt wird. Es sollte ja die Frage ganz nahe zur Hand gelegen haben: Geschieht diese Elimination in der Regel ohne eine Reizung der Nieren, oder, wenn eine solche Reizung stattfindet, ist sie ron einiger Bedeutung und kann sie vorübergehend oder für die Zukunft einen Schaden herbeiführen? Ich will versuchen, in diesem Aufsatz einen kleinen Beitrag zur Beantwortung dieser Frage zu liefern.

Es war in England, wo man zuerst beobachtete, dass Albuminurie bei Syphilispatienten auftreten kann; man schrieb indessen die Albuminurie auf Rechnung des angewendeten Quecksilbers. Gegen diese Ansicht trat Rayer (1840) auf, welcher hervorhob, dass es die syphilitische Krankheit an sich selbst sei, welche die Albuminurie hervorrufe. Seitdem hat dieses als ein Glaubensartikel gegolten, und erst im Jahre 1885 - so viel ich habe finden können - ist die Ansicht wieder ausgesprochen worden, dass die Behandlung mit Quecksilber eine zufällige Albuminurie hervorrufen könne.

Auf dem IV. Congresse für innere Medicin in Wiesbaden in dem genannten Jahre erwähnte nämlich Fürbringer, dass er bei 8 Patienten von 100, die der Mercurialbehandlung unterworfen wurden, den Harn albuminhaltig gefunden habe. Sc hu s t e r in Aachen bemerkte bei dieser Gelegenheit, dass auch er derartige Fälle beobachtet habe.

Da ich bei einer grossen Anzahl Untersuchungen von Patienten, die mit verschiedenen Quecksilberpräparaten behandelt wurden, fand, dass im Laufe der Behandlung sowohl Cylindrurie, wie Albuminurie auftreten konnte, sah ich mich 1891 für berechtigt an, in einem Aufsatz: Ueber Albuminurie und Cylindrurie durch Syphilis und Quecksilber. Nord. Med.-Arkiv, Band XXIII, Nr. 29, folgende Ansicht auszusprechen:

„,Cylindrurie und Albuminurie, auf Syphilis beruhend, sind selten in einem frühen Stadium dieser Krankheit.

In einem späteren Stadium der Syphilis tritt in einzelnen Fällen ohne entdeckbare Ursache eine besondere Form von acuter Nephritis mit Blutcylindern, Fettkorncylindern, Detritus u. s. w. zusammen mit Papulo-Tuberkeln, Gummata etc. an 
anderen Körpertheilen auf, verschwindet aber gleichzeitig mit den anderen syphilitischen Symptomen bei specifischer Behandlung bald wieder (möglicherweise kann sie auf im Zerfall begriffenen Nierensyphilomen beruhen).

Hg-Behandlung, namentlich kräftige, gibt sehr oft Anlass zu Cylindrurie, zuweilen auch zu Albuminurie, welche Leiden, gleich der Stomatitis, je nach der individuellen Disposition in schwererer oder gelinderer Form auftreten. Der Urin ohne Eiweiss und ohne Cylinder berechtigt ebensowenig wie völlig gesundes Zahnfleisch zu dem Schlusse, dass nur eine unbedeutende Menge $\mathrm{Hg}$ absorbirt worden ist. Die Kenntniss von der Grösse der absorbirten Hg-Menge wird nur durch Untersuchung des Urins oder der Foeces auf $\mathrm{Hg}$ erhalten.

Cylindrurie und Albuminurie, auf $\mathrm{Hg}$-Behandlnng beruhend, sind ziemlich schnell übergehend und lassen in der Regel keine Disposition für Nephritis für die Zukunft zurück."

Auf dem zweiten internationalen dermatologischen Congress in Wien im September 1892 hielt Schwimmer einen Vortrag: „Ueber das Vorkommen der Albuminurie bei luetischen Affectionen," in welchem er unter anderem sagte, dass er zwar bei 22 Patienten von 250, die mit $\mathrm{Hg}$ behandelt wurden, Albuminurie auftreten gesehen habe, dass er aber diese „Eiweissausscheidung als ein Symptom der constitutionellen Syphilis" betrachten mïsse und dass in dieser Hinsicht seine ,Ansichten jenen Welanders widersprechen". In der darauf folgenden Discussion hob keiner der Redner die Wahrscheinlichkeit einer Albuminuria mercurialis hervor.

Da nun Tausende von Hg-Untersuchungen uns gezeigt haben, dass eine grosse Menge $\mathrm{Hg}$ ununterbrochen durch die Nieren eliminirt wird, so sind wir ja ziemlich berechtigt zu muthmassen, dass diese Elimination eine Reizung in ihnen verursachen könne, zumal wir wissen, dass bei acuter Intoxication mit $\mathrm{Hg}$ anatomisch nachweisbare Veränderungen, wie Epithelnekrose mit oder ohne Kalkinfareten, in den Nieren auftreten können. Ullmanns zahlreiche, genaue Versuche an Thieren haben dasselbe gelehrt: "Absolut und relativ am meisten HgMetall fand sich stets in der Nierensubstanz" und dieses nicht nur bei acuten Intoxicationen, sondern ,auch bei den chronischen 
Vergiftungen sowie bei medicamentöser Dosirung, also bei relativ kleinen einverleibten $\mathrm{Hg}$-Dosen war der Metallgehalt der Niere sehr gross". Auch wenn Ullmann in diesen Fällen keine Epithelnekrose constatiren konnte, so ist eine solche doch von anderen Forschern beobachtet worden.

Auf Grund alles dieses würde es mir eigenthümlich erscheinen, wenn wir nicht auch bei Menschen bei medicamentöser Dose von $\mathrm{Hg}$ mitunter in den Nieren Zeichen einer durch die Elimination von Quecksilber hervorgerufenen Reizung beobachten könnten.

Da nun die Frage, inwiefern eine Behandlung mit $\mathrm{Hg}$ eine Reizung in den Nieren hervorrufen und nachtheilig auf sie einwirken kann, nicht nur theoretisches, sondern auch praktisches Interesse haben kann, so habe ich eine neue Serie von Untersuchungen angestellt, um meine früher ausgesprochenen Ansichten zu controliren, und es ist das Ergebniss dieser Untersuchungen, welches ich hier darlegen werde.

Will man untersuchen, ob sich eine Reizung in den Nieren vorfindet oder nicht, so genügt es nicht, mit einem mehr oder weniger empfindlichen Reagens nachzusehen, ob sich Eiweiss in nachweisbarer Menge im Harn findet oder nicht, sondern es ist nothwendig, genaue mikroskopische Untersuchungen über die Formenelemente, namentlich die Cylinder anzustellen, die im Harne rorkommen können. Dieses hat Schwimmer in seinem weiter vorn erwähnten Aufsatz unterlassen; wenigstens geschieht es nur äusserst selten, dass er etwas von den Cylindern erwähnt; es ist so gut wie ausschliesslich nur die Rede von der Albuminurie.

Dank sei Stenbeck's Erfindung des Sedimentators (der Centrifuge), so ist es nicht länger mit Schwierigkeiten verbunden, gleich nach dem Lassen des Harnes solche Untersuchungen auf Formenelemente, auf Cylinder auszuführen. Eine solche Untersuchung gibt uns über den Zustand der Nieren viel wichtigere Aufschlïsse als die Salpetersäure und auch andere, für die Albuminurie viel empfindlichere Reagentien.

In meinem weiter vorn erwähnten Aufsatz habe ich diese von allen anerkannte Auffassung auch hervorgehoben, und ich will jetzt nur einige dort vorkommende Worte von mir über 
die Bedeutung des Vorkommens und des Auftretens von Cylindern im Harne anführen. Nachdem ich betont habe, dass man auf alle Formenelemente, von denen ein Theil constant auftritt, Acht geben muss, hebe ich hervor, „dass man jedoch das grösste Gewicht auf das Vorkommen von Cylindern zu legen hat; aber da entsteht die Frage: Können Cylinder in dem Urin von einer völlig gesunden Niere vorkommen? Und dann kann man mit Bizzoze ro fragen: Gibt es wirklich eine völlig gesunde Niere? Da man weiss, dass von den Nieren beständig eine Menge für dieselben mehr oder weniger irritirender Stoffe ausgeschieden werden müssen, so könnte es nicht verwundern, wenn sich stets an irgend einer Stelle derselben ein irritativer Process fände, der sich durch eine minimale Veränderung des Harnes, z. B. durch ein paar Cylinder in demselben, zu erkennen gäbe. Um die oben gestellte Frage beantworten zu können, ist es nöthig, eine grosse Anzahl von Untersuchungen an Personen auszuführen, die mit allen den diagnostischen Mitteln, die wir jetzt haben, als völlig gesund erkannt worden sind. Solche Untersuchungen habe ich Gelegenheit gehabt, nur in einer sehr geringen Anzahl von Fällen vorzunehmen, ich glaube aber gleichwohl, dass ich auf Grund dieser und meiner an syphilitischen Personen ausgeführten Untersuchungen berechtigt bin den Schluss zu ziehen, dass man bei röllig gesunden Personen keine Cylinder antreffen darf, dass aber das Vorkommen eines oder ein paar hyaliner oder feinkörniger Cylinder uns nicht das Recht gibt, auf eine in der einen oder anderen Weise wirklich krankhafte Veränderung in den Nieren zu schliessen, die diesen Urin secernirt haben. Ganz anders gestaltet sich das Verhältniss, wenn man einen Fall verfolgt und in ihm anfänglich keine oder nur ein paar einzelne solche Cylinder findet, dieselben aber während des Verlaufes der Krankheit immer zahlreicher werden und vielleicht gar mit Blut- oder Epithelcylindern untermischt auftreten sieht. In einem derartigen Falle hat man das Recht, eine nach und nach zunehmende Reizung in den Nieren anzunehmen. Blut und Epithelcylinder und ebenso auch metamorphisirte $\mathrm{Cy}$ linder haben ja ihre Bedeutung, aber auch eine grosse Anzahl hyaliner und feinkörniger Cylinder dürfte sicher einen mehr 
oder weniger abnormen Zustand in der Niere zu erkennen geben."

Diese meine damals ausgesprochenen Ansichten stimmen vollkommen mit denen überein, zu welchen ich auf Grund der zahlreichen Untersuchungen gekommen bin, die ich seit jener Zeit ausgeführt habe.

Wenn zur Untersuchung Harn von einer Frau angewendet wird, so ist er mittelst des Katheters zu nehmen; wird der Harn von einem Mann genommen, so ist der zuerst gelassene Theil desselben zur Sedimentirung nicht anzuwenden, weil sich in ihm Trippereiter, Tripperfäden u. dgl. finden können, worin die Untersuchung auf Cylinder auf mehr oder weniger bedeutende Hindernisse stossen kann.

Wenn ich nun die Ergebnisse dieser meiner letzten Untersuchungen ïber Cylindrurie und Albuminurie, durch Behandlung mit $\mathrm{Hg}$ verursacht, darlege, so scheint es mir am zweckmässigsten zu sein, erst über das Auftreten der Hg-Cylindrurie $\mathrm{zu}$ berichten.

Das Vorkommen und Auftreten von Cylindern im Harn kann selbstverständlich keine Bedeutung als Beweis dafür haben, dass die Cylinder durch eine Behandlung mit Quecksilber hervorgerufen sind, sofern es sich nicht zeigt, dass sie in der Regel in dem Verhältniss auftreten, an Menge zunehmen und sich vielleicht auch in ihrer Beschaffenheit entwickeln, in dem die Behandlung mit $\mathrm{Hg}$ fortschreitet. Sollten die Cylinder in der Syphilis ihren Grund haben, so müsste ja ihr Auftreten und Verschwinden mit dem Auftreten und Verschwinden der syphilitischen Symptome in Zusammenhang stehen; stehen sie dagegen mit der Behandlung mit Quecksilber, d. h. der Elimination des Quecksilbers durch die Nieren in Zusammenhang, so muss ihr Auftreten und Verschwinden auch mit einer vermehrten oder verminderten Ausscheidung von Quecksilber durch die Nieren Uebereinstimmung zeigen.

Haben also die Cylinder in der Syphilis ihren Grund, so dürfte man sie im Anfange der Behandlung finden und sie dann gleichzeitig mit den übrigen syphilitischen Symptomen verschwinden sehen; haben sie dagegen in der Behandlung mit $\mathrm{Hg}$ ihren Grund, so sollte man im Anfange der Behandlung: 
keine oder nur einzelne Cylinder, einen mehr oder weniger bedeutenden Cylindergehalt aber erst am Schlusse der Behandlung finden.

In meinem vorigen Aufsatz habe ich erwähnt, dass ich von 15 Patienten, die ich für den ersten Ausbruch der Syphilis behandelte, im Anfange der Behandlung bei 13 keinen einzigen Cylinder entdecken konnte und nur bei 2 ein paar hyaline Cylinder antraf. Von 11 Patienten, welche ihre Behandlung für Recidiv (secundäres Stadium) begannen, konnte ich Cylinder nur bei einem entdecken, und auch er hatte nur ein paar. Von 13 wegen tertiären Symptomen in das Krankenhaus aufgenommenen Patienten hatten 10 keine Cylinder, 2 einige und einer ziemlich viel hyaline und feinkörnige. Also von 39 Patienten in verschiedenen Stadien der Syphilis hatten 33 keine Cylinder, und ron den 6, bei denen Cylinder gefunden wurden, hatten 5 nur ein paar hyaline Cylinder, d. h. nicht mehr, als man bei röllig gesunden Personen antreffen kann, und nur einer hatte eine grössere Anzahl, was ja alles dagegen spricht, dass die Syphilis an und für sich Cylindrurie verursacht.

Ich habe nun Gelegenheit gehabt, 97 Patienten vor dem Beginn der Behandlung zu untersuchen. Bei 17 von ihnen, d. h. bei 17.5 Proc. waren Cylinder zu entdecken (welcher Procentsatz ziemlich nahe mit dem in meiner vorigen Serie erhaltenen zusammenfällt, wo er 15.4 Procent betrug). Auch diese $\mathrm{Pa}$ tienten haben sehr verschiedene Symptome von Syphilis, wie Sclerosis, Roseola, papulöses und pustulöses Syphilid, Gummata, Knochenaffection, Gehirn- und Rückenmarkssyphilis u. s. w. gehabt. Von 46 Patienten mit dem ersten Ausbruch der Syphilis haben 6, von 36 mit Recidiv (secundärem Stadium) 5 und ron 15 mit tertiären Symptomen 6 Cylinder gehabt. Von diesen Patienten, bei denen sich Cylinder fanden, haben sieben 1 Cylinder, ${ }^{1}$ ) vier 2 und einer 3 Cylinder gehabt; vier haben einzelne Cylinder und nur einer hat Cylinder hier und

1) Wenn ich z. B. „einen hyalinen Cylinder" angebe, so meine ich damit, dass ich trotz sorgfältigen Suchens in 1 oder 2 Präparaten nicht mehr als diesen einen Cylinder habe finden können; ich habe selbstver. ștändlich nicht das Sediment der ganzen Harnmenge untersucht. 
da gehabt. Der Cylindergehalt bei den ersten 12 Patienten ist mithin nicht grösser gewesen als derjenige, der bei völlig gesunden Personen vorkommen kann, und bei den anderen 5 hat er sich nur unbedeutend, wenn überhaupt etwas grösser als bei diesen gezeigt. Von 5 Patienten hatten 4 Albuminurie bei ihrer Aufnahme in das Krankenhaus, und bei einem stellte Albuminurie sich im Laufe der Behandlung ein. Es scheint mir am zweckmässigsten zu sein, diesen letzten Fall näher zu besprechen, wenn ich über Albuminuria mercurialis berichte. (Siehe Fall XX.)

Auch diese Serie spricht ja bestimmt gegen die Annahme, dass die Syphilis an und für sich selbst, anders als ausnahmsweise, Cylindrurie verursacht.

Wir wissen nun, dass bei der Behandlung mit Einreibungen, Ueberstreichungen und Einspritzungen ron löslichen und unlöslichen Hg-Salzen eine ziemlich schnelle und gleichmässige Absorption ron $\mathrm{Hg}$ stattfindet, was sich durch eine constant während der ganzen Dauer der Behandlung steigende Elimination von $\mathrm{Hg}$ zu erkennen gibt; eine beträchtliche Elimination von $\mathrm{Hg}$ ist noch einige Zeit nach Abschluss der Behandlung zu beobachten; später nimmt die Menge des eliminirten $\mathrm{Hg}$ mehr und mehr ab.

Ist es nun so, dass die Behandlung mit $\mathrm{Hg}$, d. h. die Elimination von $\mathrm{Hg}$ durch die Nieren, in den Nieren eine Reizung hervorrufen kann und dass diese Reizung sich durch das Auftreten von Cylindern im Harn zu erkennen gibt, so dürften wir ja berechtigt sein zu erwarten, dass ein Cylindergehalt im Harn im Anfange der Behandlung entweder gar nicht oder in nur minimaler Grösse vorhanden ist, dass später aber Cylinder auftreten und, je nachdem die Menge des eliminirten Quecksilbers wächst, immer zahlreicher werden, was mit anderen Worten sagen will, dass wir im Laufe der Behandlung Cylinder auftreten und sich dann allmälig bis zum Schlusse der Behandlung vermehren sehen.

Ich will hier den Raum nicht mit der Aufzählung einer Menge von Fällen füllen, die ich in dieser Richtung untersucht labe, sondern ich begnüge mich damit, nur folgende an- 
zuführen, welche zeigen, wie der Cylindergehalt während der Behandlung wächst.

\section{Erster Ausbruch der Syphilis.}

Fall I. B., 26 Jahre alt, wurde am 9./9. in das Krankenhans aufgenommen. Beliandlung mit Sozojodol-Hg-Einspritzungen.

10./9. 1 Einspr.; kein Alb.; 1,020 spec. Gew.; keine Cylinder.

15.9. $2 "$ " " $1,025 "$ " keine Cylinder.

20.9. $3 "$ " " " $" 1,023 "$ " $"$ einzelne Cylinder.

25. $9.4 " \quad " 1,016 "$ " $"$ hie und da Cylinder.

30./9. 5 " " " $" 1,018, "$ ziemlich viel Cylinder.

$6.110 .6 " \quad " \quad " 1,019 " n$ " viel Cylinder.

11./10.7 " " $7,018, \quad$ " sehr viel Cylinder.

Fall II. E., 21 Jahre alt, wurde in das Krankenhaus am 13./9. aufgenommen. Behandlung mit Sozojodol-Hg-Finspritzungen.

19./9. 1 Einspr.; 20./9. Kein Alb.; ? spec. Gew.; keine Cylinder.

\begin{tabular}{|c|c|c|c|c|c|c|c|c|}
\hline $24.9 . \quad 2$ & & $25 . / 9$ & & & 1,025 & " & " & einzelne Cylinder. \\
\hline 29.9 . & " & 29.9. & " & $"$ & 1,020 & भ & $"$ & zieml. viel Cylinder. \\
\hline $4 . / 10.4$ & $n$ & $5 . / 10$. & $"$ & $"$ & 1,020 & $n$ & $n$ & hie u. da Cylinder. \\
\hline $9 . / 10.5$ & $"$ & 10./10. & $"$ & $"$ & 1,017 & $m$ & $"$ & recht viel Cylinder. \\
\hline $14 . / 10.6$ & $"$ & $15 . / 10$. & $n$ & $n$ & 1,011 & $"$ & $"$ & sehr viel Cylinder. \\
\hline 19./10. 7 & $"$ & $20 . / 10$. & 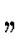 & $m$ & 1,015 & $n$ & $"$ & sehr viel Cylinder. \\
\hline
\end{tabular}

Recidiv (secundäres Stadium).

Fal1 III. S., 38 Jahre alt, wurde in das Krankenhaus am 5./6. aufgenommen. Behandlung mit Sozojodol-Hg-Einspritzungen.

6./6. 1 Einspr.; kein Alb.; 1,016 spec. Gew.; keine Cylinder.

11.6. $2 " \quad " \quad, 1,020, \quad$ zwei Cylinder.

16.6. $3 "$ " $" 1,019 "$ ziemlich viel Cylinder.

21./6. $4 " n " n, 1,017 n$ ziemlich viel Cylinder.

26.6. 5 $5 \quad " \quad$ " $\quad 1,020 \% \quad$ viel Cylinder.

1./7. $6 "$ " $" 1,017 "$ viel Cylinder.

6./7. $7 " \# " 1,022 " n$ Cylinder in bedeut.Menge.

Fall IV. H., 28 Jahre alt, wurde in das Krankenhaus am 11./9. aufgenommen. Behandlung mit Ueberstreichungen von $: 6 \mathrm{Gr}$. Ung-Hg.

12./9. - $\quad$ kein Alb.; 1,027 spec. Gew.; keine Cylinder.

17./9. 5 Ueberstr.; " ", $025 "$ " einzelne Cylinder.

22./9. $10 " \# " 1,028 "$ " $"$ einzelne Cylinder.

27.9. $15 " \quad " \quad " 1,025 "$ " $"$ hie und da Cylinder.

2.10. $20 \quad " \quad " \quad " 1,026 \quad " \quad$ " viel Cylinder.

7./10. $25 \quad " \quad " \quad " 1,016 \quad " \quad$ " sehr viel Oylinder.

12./10. $30 " n ", \quad ", 022 " n$ sehr viel Cylinder.

Recidiv (tertiäres Stadium).

Fall V. W., 45 Jahre alt, wurde in das Krankenhaus am 3./5. aufgenommen. Behandlung mit Ueberstreichungen von $6 \mathrm{Gr}$. Ung-Hg. 
5./5. 1 Ueberstr.; kein Alb.; keine Cylinder.

\begin{tabular}{|c|c|c|c|c|c|}
\hline $15 . / 5$. & 11 & $n$ & $"$ & \% & hie und da Cylinder. \\
\hline $20 . / 5$ & 16 & & & & recht viel Cylinder. \\
\hline $25 . / 5$. & 21 & $\eta$ & 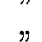 & " & eine Menge Cylinder. \\
\hline $30 . / 5$. & 26 & $"$ & 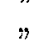 & $\eta$ & eine Menge Cylinder. \\
\hline 4. $/ 6$ & 31 & $n$ & $\eta$ & $n$ & Cylinder in bedeutender Menge. \\
\hline & 30 & $n$ & $\eta$ & $\eta$ & Cylinder in bedeutender Menge. \\
\hline
\end{tabular}

In diesen wie in einer Menge anderen Fällen ist der Cylindergehalt sehr gestiegen und bei Schluss der Behandlung bedeutend gewesen; aber auch da, wo dieses nicht der Fall gewesen ist, hat eine stetige Vermehrung der Cylinder, obschon in einem geringeren Grade wie in den angeführten Fällen, stattgefunden. Als Beispiel will ich folgenden Fall anführen:

Fall VI. 0., 3ă Jahre alt, wurde in das Krankenhaus am 16./9. aufgenommen. Behandlung mit Einspritzungen von Sozojodol-Hg.

17.9. 1 Einspr.; kein Alb.; 1,024 spec. Gew.; zwei kleine Cylinder.

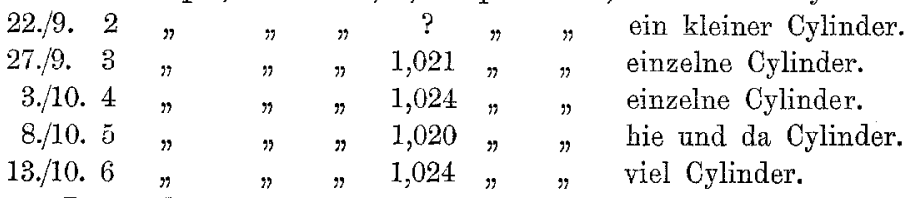

In noch geringerem Grade vermehrten sich die Cylinder in folgendem Fall:

Fall VII. M., 25 Jahre alt, wurde in das Krankenhans am 11./9. aufgenommen. Behandlung mit Ueberstreichungen von $6 \mathrm{Gr}$. Ung-Hg.

13./9. - $\quad$ kein Alb.; 1,020 spec. Gew.; keine Cylinder.

18./9. 5 Ueberstr.; " " $1,025 "$ " zwei Cylinder.

23.9. $10 " \quad " \quad \Rightarrow 1,023 " \Rightarrow \quad$ " keine Cylinder.

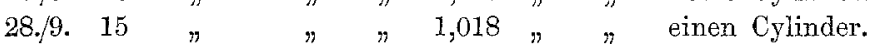

3.110. $20 " \quad " \quad " 1,025 " \quad "$ " $"$ hie und da Cylinder.

9./10. $26 "$ " " ? " " $"$ ziemlich viel Cylinder.

Schliesslich finden sich Fälle, wo der Gehalt an Cylindern so wenig gewachsen ist, dass er sich bei Schluss der Behandlung innerhalb des normalen Gebietes befunden hat, und ebenso gibt es Fälle, wo es während der ganzen Behandlung nicht möglich gewesen ist, einen einzigen Cylinder zu entdecken, z. B. in folgenden beiden Fällen.

Fall VIII. W., 31 Jahre alt, wurde in das Krankenhaus am 15./7. aufgenommen. Behandlung mit Sozojodol-Hg-Einspritzungen.

17./7. 1 Einspr.; kein Alb.; ? spec. Gew.; keine Cylinder.

22./7. $2 "$ " ? " " keine Cylinder.

27.7. 3 " " " $" 1,010 "$ " keine Cylinder.

1./8. 4 " " " 1,015 " " " "

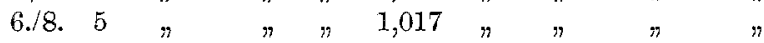


Fall IX. F., 40 Jahre alt, wurde in das Krankenbaus am 15./8. aufgenommen. Bebandlung mit Einreibungen und Ueberstreichungen von je drei Gr. Ung.-Hg.

16./8. keine Einr. u. Ueberstr.; kein Alb.; 1,025 spec. Gew.; keine Cyl.

\begin{tabular}{|c|c|c|c|c|c|c|c|c|c|}
\hline 21./8. 5 & $"$ & , & $n$ & " & 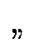 & 1,027 & $"$ & $n$ & $n$ \\
\hline $26 . / 8, \quad 10$ & $\eta$ & " & $"$ & $"$ & $n$ & 1,022 & $"$ & $n$ & $n$ \\
\hline $31 . / 8 . \quad 15$ & $"$ & 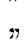 & $"$ & $"$ & $"$ & 1,019 & $n$ & $"$ & $"$ \\
\hline $11 . / 9$ & $"$ & " & $"$ & $"$ & $"$ & 1,015 & $"$ & $"$ & $n$ \\
\hline $16 . / 9$ & $\eta$ & $n$ & $"$ & $n$ & $n$ & 1,015 & $n$ & $"$ & $n$ \\
\hline 21./9. & $"$ & " & $"$ & $"$ & $"$ & $?$ & $\eta$ & $n$ & drei \\
\hline $25 . / 9 . \quad 40$ & ", & . & 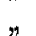 & " & $n$ & 1,020 & " & " & einen \\
\hline
\end{tabular}

2./10. Der Patient hatte mit der Behandlung 6 Tage ausgesetzt, nimmt sie heute aber wieder anf.

2./10. 40 Einr. u. Ueberstr.; kein Alb.; 1,005 spec. Gew.; keine Cyl.

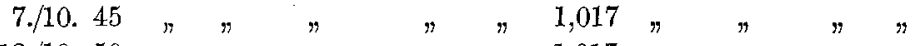

12.10. $50 \quad " \quad " \quad " \quad " 1,017, \quad " \quad$ " $"$

In einem Theil der Fälle hat der Cylindergehalt kein solches regelmässiges Steigen wie in den oben angeführten Fällen I-V gezeigt. Es ist vorgekommen, dass ein Patient bei einer Untersuchung einen etwas geringeren Cylindergehalt als bei der vorhergegangenen gehabt hat, aber wo dieses geschehen ist, ist die Verminderung im Cylindergehalt stets nur eine unbedeutende gewesen; es ist z. B. niemals vorgekommen, dass ein Patient, bei dem das eine Mal eine Menge Cylinder gefunden wurden, das nächste Mal keine oder nur einzelne Cylinder gehabt hat, wohl ist es aber zuweilen geschehen, dass ein Patient, bei dem bei einer Untersuchung Cylinder in grosser Menge gefunden wurden, bei der nächsten Untersuchung eine geringere aber doch reichliche Menge Cylinder gehabt hat. Mitunter kann dieses auf der Concentration des Harns beruht haben; so kann ein Patient an dem einen Tage, wo der Harn von normaler Concentration ist, eine ziemlich grosse Menge Cylinder haben, während er am Tage darauf, wenn er viel Wasser getrunken und sein Harn ein sehr geringes spec. Gewicht besitzt, Cylinder nur hie und da hat. Solche Fälle sind zwar ziemlich selten, doch kommen sie vor. Die allgemeine Regel ist die, dass der Cylindergehalt constant mehr oder weniger steigt, so lange die Behandlung mit $\mathrm{Hg}$ dauert.

Wenn man nun berechtigt sein soll zu behaupten, dass die Hg-Ausscheidung in der Regel eine gewisse Reizung auf 
die Nieren ausübt, so muss man auch zeigen können, nicht nur dass der Cylindergehalt in der grossen Mehrzahl der Fälle sich vermehrt, sondern auch, dass er sich in dem Grade vermehrt, dass er bei Schluss der Behandlung mehr oder weniger bedeutend grösser ist als bei Beginn derselben und bei gesunden Personen. Folgendes Tableau zeigt, wie gross der Cylindergehalt bei Beginn und bei Schluss der Behandlung bei 100 Personen gewesen ist; es zeigt auch, wie gross der Cylindergehalt bei Schluss der Behandlung bei 42 anderen Personen gewesen ist, die ich nicht bei Beginn der Behandlung, wohl aber bei Schluss derselben und zum grossen Theil auch ein paar Mal während der Behandlung untersucht habe.

Tabelle I.

\begin{tabular}{|c|c|c|c|c|c|c|c|c|c|c|c|}
\hline \multirow{3}{*}{ 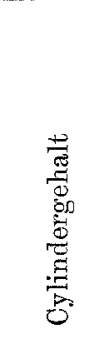 } & \multicolumn{5}{|c|}{ 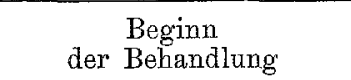 } & \multicolumn{5}{|c|}{ der $\begin{array}{l}\text { Sehluss } \\
\text { Behandlung }\end{array}$} & \multirow[b]{2}{*}{ 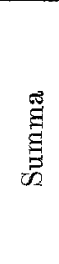 } \\
\hline & 离 & 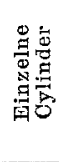 & 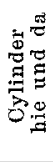 & 离 & 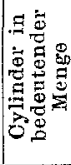 & 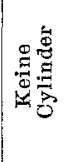 & 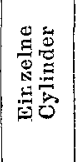 & 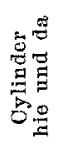 & 兽 & 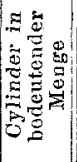 & \\
\hline & $\begin{array}{l}83 \\
-\end{array}$ & 16 & 1 & - & - & $\begin{array}{l}3 \\
3 \\
\end{array}$ & $\begin{array}{l}17 \\
10\end{array}$ & $\begin{array}{l}30 \\
11\end{array}$ & $\begin{array}{r}17 \\
4 \\
\end{array}$ & $\begin{array}{l}33 \\
14\end{array}$ & $\begin{array}{r}100 \\
42 \\
\end{array}$ \\
\hline Summa & 83 & 16 & 1 & - & - & 6 & 27 & 41 & 21 & 47 & 142 \\
\hline Proc. & 83 & 16 & 1 & - & - & 4,2 & 19,0 & 28,9 & 14,8 & 33,1 & - \\
\hline
\end{tabular}

Wir finden also, dass bei 23.2 Procent keine augenscheinliche Vermehrung der Cylinder stattgefunden hat, dass bei 28.9 Procent die Vermehrung der Cylinder nicht unbedeutend und bei 47.9 Procent sehr bedeutend gewesen ist. Dieses stimmt ziemlich gut mit den Ergebnissen meiner vorigen Untersuchungsserien überein, wo bei 30 Procent der Cylindergehalt bei Schluss der Behandlung gering oder minimal warbei den übrigen 70 Procent zeigte sich aber auch dort eine mehr oder weniger bedeutende Vermehrung des Cylindergehaltes. Alles dieses, so scheint es mir, kann nicht anders gedeutet werden, als dass die Vermehrung des Cylindergehaltes in der Hg-Elimination durch die Nieren ihren Grund hatte, daher ich glaube behaupten zu können, dass es sehr oft, vielleicht in der Regel eintrifft, dass die Hg-Behandlung eine 
mehr oder weniger grosse Reizung auf die Nieren aus dem Grunde ausuibt, dass ein grosser Theil des während der Behandlung in den Organismus gekommenen Quecksilbers durch sie ausgeschieden wird.

Ist dieses nun wirklich der Fall, so dürften wir erwarten können, dass nach Schluss der Behandlung mit Hg der Cylindergehalt auch abzunehmen beginnt. Aber die Grösse der Hg-Ausscheidung nimmt nicht gleich nach dem letzten Behandlungstage $a b$; namentlich ist dieses bei der Einspritzung einer grösseren Menge $\mathrm{Hg}$ auf einmal, z. B. bei der Einspritzung von Thymol-Hg der Fall. Hier vermehrt sich die Absorption und Elimination noch eine kurze Zeit nach dem letzten Einspritzungstage, um sich dann allmählich zu vermindern. Unter allen Umständen kann man die Ausscheidung von $\mathrm{Hg}$, wenn sie bei Schluss der Behandlung gross ist, auch noch einige Zeit nachher gross finden, worauf sie dann nach und nach ziemlich schnell abnimmt. Da frägt man sich selbstverständlich: Finden sich entsprechende Veränderungen in der Grösse des Cylindergehaltes? Da die meisten Patienten einen oder ein paar Tage nach dem Abschluss der Behandlung mit $\mathrm{Hg}$ aus dem Krankenhause entlassen werden, so ist es beinahe ganz unmöglich, in dieser Richtung einige Untersuchungen ausführen zu können. Durch einen Zufall habe ich jedoch Gelegenheit erhalten, in den folgenden zwei Fällen eine vollständige SerienUntersuchung auch nach Abschluss der Behandlung auszuführen. Diese Fälle scheinen mir so beweisend zu sein, dass ich sie in extenso anführen will.

Fall X. B., 25 Jahre alt, wurde in das Krankenhaus am 21./8. aufgenommen. Behandlung mit Thymol-Hg-Einspritzungen.

25./8. 1 Einspr.; kein Alb.; 1,020 spec. Gew.; keine Cylinder.

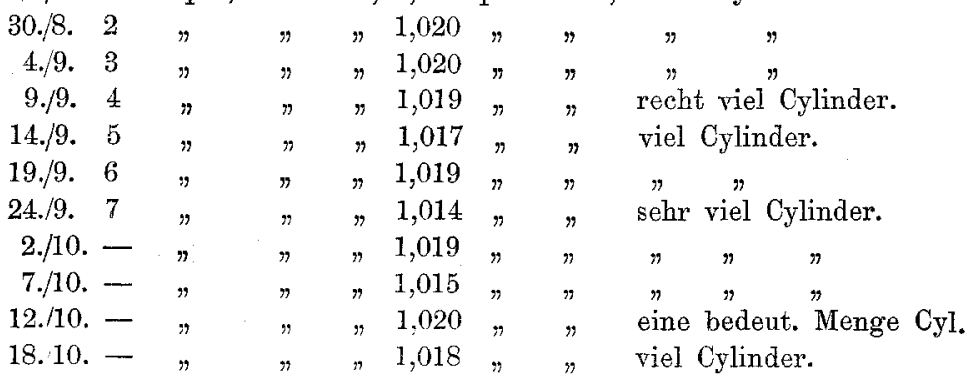


24./10. - Einspr.; kein Alb.; 1,020 spec. Gew.; ziemlich viel Cylinder.

\begin{tabular}{|c|c|c|c|c|c|c|c|}
\hline 29./10. - & "7 & & $\eta$ & 1,021 & $n$ & $n$ & hie und da Cylinder. \\
\hline $3 . / 11$. - & $\eta$ & & $n$ & 1,023 & $n$ & $"$ & einzelne Cylinder. \\
\hline 8./11. - & $"$ & & $"$ & 1,022 & $"$ & $n$ & drei Cylinder. \\
\hline 13./11. - & $n$ & $n$ & " & 1,021 & 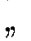 & $m$ & keine Cylinder. \\
\hline 18./11. - & , & " & m & 1,023 & $"$ & " & $"$ \\
\hline
\end{tabular}

Fall XI. S., 47 Jahre alt, wurde in das Krankenhaus am 8./9. aufgenommen. Behandilung mit Ueberstreichungen von $6 \mathrm{Gr}$. Ung.-Hg.

8.9. Keine Ueberstr.; kein Alb. Salp.; kein Alb. Trichlor-Essigs.; 1,024 spec. Gew.; ein Cylinder.

13./9. 5 Ueberstr.; kein Alb. Salp.; kein Alb. Trichlor-Essigs.; 1,025 spee. Gew.; fünf Cylinder.

18.19. 10 Ueberstr.; kein Alb. Salp.; kein Alb. Trichlor-Essigs.; 1,023 spec. Gew.; hie und da Cylinder.

23./9. 15 Ueberstr.; kein Alb. Salp.; kein Alb. Trichlor-Essigs.; 1,014 spec. Gew.; viel Cylinder.

28./9. 20 Ueberstr.; kein Alb. Salp.; kein Alb. Trichlor-Essigs.; 1,017 spec. Gew.; viel Cylinder.

3.10, 26 Ueberstr.; kein Alb. Salp.; Spuren von Alb. Trichlor-Essigs.; 1,015 spec. Gew.; eine bedeutende Mienge Cylinder.

8./10. 30 Ueberstr.; Spuren von Alb. Salp.; Alb. Trichlor-Essigs.; 1,012 spec. Gew.; eine bedeutende Menge Cylinder.

13./10. - Ueberstr.; Alb. Salp.; Albumin Trichlor-Essigs.; 1,019 spec. Gew.; eine sehr bedeutende Menge Cylinder.

18./10. - Ueberstr.; Spuren von Alb. Salp.; Alb. Trichlor-Essigs.; 1,017 spec. Gew.; eine sehr bedeutende Menge Cylinder.

23./10. - Ueberstr.; kein Alb. Salp.; Albumin Trichlor-Essigs.; 1,013 spec. Gew.; sehr viel Cylinder.

28./10. - Ueberstr.; kein Alb. Salp.; Spuren von Alb. Essigs.; 1,016 spec. Gew.; viel Cylinder.

2./11. - Veberstr.; kein Alb. Salp.; unbed. Spuren von Alb. Essigs.; 1,016 spec. Gew.; recht viel Cylinder.

7./11. - Ueberstr.; kein Alb. Salp.; unbed. Spuren von Alb. Essigs.; 1,016 spec. Gew.; recht viel Cylinder.

12./11. - Ueberstr.; kein Alb. Salp.; unbed. Spuren ron Alb. Essigs.; ? spec. Gew.; ziemlich viel Cylinder.

17./11. - Ueberstr.; kein Alb. Salp.; unbed. Spuren von Alb. Essigs.; 1,020 spec. Gew.; einzelne Cylinder.

In diesen beiden Fällen ist ja die Uebereinstimmung zwischen der $\mathrm{Hg}$-Elimination und dem Cylindergehalt schlagend. Ob sie es immer ist, weiss ich nicht; dass sie es aber im Grossen. gewesen ist, habe ich auf Grund von Untersuchungen, die ich zufälligerweise das eine oder das andere Mal bei Patienten eine kürzere oder längere Zeit nach Schluss der Behandlung aus- 
Kann d. Behandl. mit Quecks.Cylindrurie u. Albuminurie hervorrufen? 345

führen konnte, alle Ursache anzunehmen. Als Beweis hierfür will ich folgende Tabelle anführen, die das Steigen des Cylindergehaltes während der Behandlung sowie seine Verminderung oder sein Verschwinden kürzere oder längere Zeit nach Schluss derselben zeigt.

Tabelle II.

\begin{tabular}{|c|c|c|c|c|c|c|}
\hline \multirow{2}{*}{\multicolumn{2}{|c|}{ 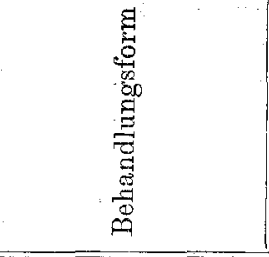 }} & \multicolumn{4}{|c|}{ Cylindergehalt } & \multirow{19}{*}{$\begin{array}{l}\text { Th.-Hg-Einspr. = Einsprit- } \\
\text { zung von essigsaurem } \\
\text { Thymol-Quecksilber. } \\
\text { Soz.-Hg-Einspr. = Einsprit- } \\
\text { zung von Sozojodol- } \\
\text { Quecksilber. }\end{array}$} \\
\hline & & 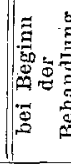 & 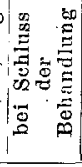 & 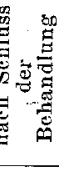 & 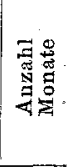 & \\
\hline & Th.-Hg-Einspr. & 0 & 3 & 1. & 1 & \\
\hline & Ueberstr. & 0 & 3 & 2 & 1 & \\
\hline 35 & & 0 & 4 & 1 & $11 / 4$ & \\
\hline & Th:-Hg-Einspr. & 0 & 3 & 1 & $11 / 4$ & \\
\hline & Soz.-Hg- & 0 & 2 & 0 & $11 / 4$ & \\
\hline & $n "$ & 0 & -3 & 0 & $11 / 4$ & \\
\hline & Th.-Hg- & 0 & 2 & 0 & $11 / 2$ & \\
\hline & Ueberstr. & 1 & 4 & 0 & $12 \%$ & \\
\hline & Soz.-Hg-Einspr. & 1 & 4 & 0 & $12 / 3$ & \\
\hline & Ueberstr. & $?$ & 4 & 1 & $21 / 4$ & \\
\hline 35 & $n$ & 0 & 4 & 0 & $2 / 3$ & \\
\hline 31 & $n$ & 0 & 3 & 0 & $21 / 2$ & \\
\hline 35 & $"$ & 0 & 4 & 0 & $21 / 2$ & \\
\hline 39 & $"$ & 0 & 3 & 0 & $2 \frac{1 / 2}{12}$ & \\
\hline 30 & 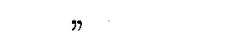 & 0 & 4 & 0 & $2 \%$ & \\
\hline 50 & $n$ & $?$ & 4 & 0 & $23 / 4$ & \\
\hline 25 & $"$ & 0 & 4 & 0 & $31 / 4$ & \\
\hline
\end{tabular}

Wenn der Cylindergehalt nach Schluss der Behandlung allmälig abnimmt, so sollten wir ja bei Aufnahme eines Patienten in das Krankenhaus wegen eines Recidivs keine oder kaum einige Cylinder antreffen. Dieses ist auch der Fall, was aus folgender Tabelle hervorgeht, in der die Fälle angeführt sind, in denen es constatirt werden konnte, wann der Patient das letzte Mal Symptome gehabt hatte und mit $\mathrm{Hg}$ behandelt worden war. 
Tabelle III.

\begin{tabular}{|c|c|c|c|c|c|c|c|c|c|c|}
\hline \multirow{3}{*}{$\begin{array}{l}\text { Anzahl Jahre und } \\
\text { Monate seit der } \\
\text { letzten Behandlung } \\
\text { mit Hg }\end{array}$} & \multicolumn{10}{|c|}{ C y lindergehalt } \\
\hline & \multicolumn{5}{|c|}{$\begin{array}{c}\text { bei der Aufnahme in das } \\
\text { Krankenhaus }\end{array}$} & \multicolumn{5}{|c|}{$\begin{array}{c}\text { bei Sehluss der Behandlung } \\
\text { mit } \mathrm{Hg}\end{array}$} \\
\hline & 题 & 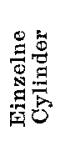 & 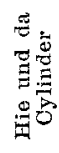 & 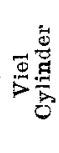 & 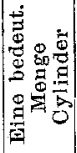 & 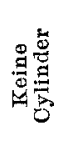 & 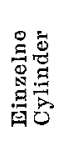 & 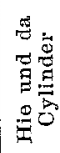 & 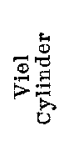 & 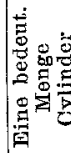 \\
\hline Ueber 1 Jahr & 8 & 1 & - & - & - & - & - & 2 & 2 & 5 \\
\hline $1 / 2-1$ Jahre & 2 & - & 一 & - & - & - & - & - & - & 2 \\
\hline $3-6$ Monate & 4 & - & 一 & - & - & 一 & - & 3 & 一 & 1 \\
\hline $2-3 \quad n$ & 6 & $\longrightarrow$ & 一 & - & - & - & 1 & - & 1 & 4 \\
\hline $1-2$ & 9 & 一 & - & - & 一 & - & 2 & 2 & 2 & 3 \\
\hline
\end{tabular}

Wir sehen, dass 29 von 30 Patienten bei ihrer Wiederaufnahme in das Krankenhaus keine Cylinder hatten. Dass diese Patienten gleichwohl für die $\mathrm{Hg}$-Elimination sehr empfindlich waren, zeigt die grosse Anzahl derselben (50 Proc.), die bei Schluss der Behandlung mit Hg. eine bedeutende Menge Cylinder bekommen hatten. Mit grösster Wahrscheinlichkeit können wir annehmen, das ssie auch bei Schluss ihrer vorigen Behandlung einenje nach der Stärke der Behandlung — grösseren oder kleineren Cylindergehalt gehabt haben.

Wie lange es währt, bis der Cylindergehalt verschwunden ist, beruht sehr wahrscheinlich auf der grösseren oder geringeren Menge Cylinder, die sich bei Schluss der Behandlung finden, sowie auf der grösseren oder geringeren Menge $\mathrm{Hg}$, das bei der Behandlung angewendet worden ist. Alles scheint. mir dafür zu sprechen, dass der Cylindergehalt viel schneller verschwindet, als die $\mathrm{Hg}$-Elimination. Diese, das wissen wir ja, fährt noch ziemlich lange fort, obschon sie einige Wochen nach Abschluss der Behandlung nur in sehr kleinen Mengen geschieht, so klein, dass sie nicht länger eine Reizung auf die Nieren ausüben können.

In der Regel ist der Cylindergehalt nach 4-6 Wochen nach Schluss der Behandlung verschwunden, doch beobachte ich gegenwärtig einen Fall, wo noch eine bedeutende Cylindrurie 10 Wochen nach beendigter Behandlung vorhanden ist. 
Wenn nun die Cylindrurie auf der Quecksilberelimination und nicht auf der Syphilis an und für sich beruht, so liesse es sich ja erwarten, dass der Cylindergehalt in directer Proportion zur Quecksilberelimination, aber in indirecter zu den auftretenden syphilitischen Symptomen stehen dürfte, d. h. dass bei Schluss der Behandlung, wenn die syphilitischen Symptome verschwunden sind und die Hg-Elimination gross ist, der Cylindergehalt sich als gross erweisen dürfte; wenn dagegen ein höchst bedeutender Theil des $\mathrm{Hg}$ eliminirt worden ist und ein Recidiv, neue syphilitische Symptome aufzutreten beginnen, dürfte sich ein minimaler oder gar kein Cylindergehalt finden. Dass es sich so verhält, dafür sprechen ja die obigen Tabellen II-III. In einzelnen Fällen ist dieses übrigens allzu augenscheinlich gewesen, um unrichtig aufgefasst werden zu können. Von solchen Fällen will ich hier nur den folgenden anführen:

Fall XII. S., 36 Jahre alt, wurde mit Ueberstreichungen wegen Rupia syphilitica behandelt.

13./4. 40 Ueberstr.; kein Alb.; eine höchst bed. Menge Cyl.; geheilt.

23./4. $50 " \quad " \quad$ " eine kolossale Menge Cylinder. Symptomfrei aus dem Krankenhause entlassen.

13./7. wurde der Patient von Neuem wegen pustulösem Syphilid mit grossen Geschwüren in das Krankenhaus aufgenommen. Behandlung mit Ueberstreichungen.

13./7. keine Ueberstr.; kein Alb.; 1,020 spec. Gew.; keine Cylinder.

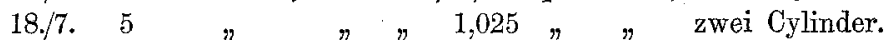

23./7. $10 " \quad " \quad " \quad 1,025 "$ " $" \quad$ einzelne Cylinder.

28./7. $15 "$ " $" 1,025 "$ " hie und da Cylinder.

2./8. $20 \quad " \quad " \quad$ " $1,020 "$ " $\quad$ zieml. viel Cylinder.

7./8. $25 " \Rightarrow " 1,024 " n$ viel Cylinder.

12./8. $30 \quad " \quad " \quad " 1,020 " n \quad " \quad$ sehr viel Cylinder.

17./8. 35 " " " $1,021, " \quad " \quad "$

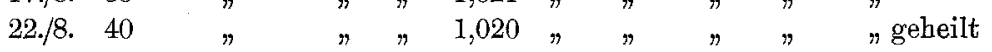

27./8. 45 "Spuren v. Alb.; 1,015 " " " " " "

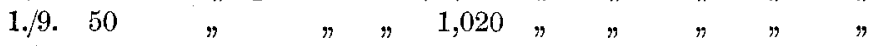

6./9. - wurde frei von "Symptomen aus dem "Krankenhause entlassen.

18./10. wurde er für Rupia syphilitica wieder in das Krankenhaus aufgenommen. Behandlung mit Jodkalium.

19./10. keine Ueberstr.; kein Alb.; 1,017 spec. Gew.; ein Cylinder.

23./10. " " " " $\quad$ ", 024 " vier Cylinder. Den 24./10. Ueberstreichungen mit Ung. Hg. 
29./10. 5 Ueberstr.; kein Alb.; 1,022 spec. Gew.; hie und da Cylinder.

\begin{tabular}{|c|c|c|c|c|c|c|c|}
\hline $3 . / 11.10$ & $"$ & & $"$ & 1,024 & $"$ & $"$ & $" \quad " \quad n \quad " \quad$ \\
\hline $8 . / 11.15$ & $"$ & & $\eta$ & 1,029 & $"$ & $n$ & ziemlich viel Cylinder. \\
\hline 13./11. 20 & $\eta$ & & $\%$ & 1,012 & $n$ & $"$ & sehr viel Cylinder. \\
\hline $18 . / 11.25$ & $"$ & Spuren & v. Alb.; & 1,017 & $n$ & $"$ & $"$ geheilt. \\
\hline $23 . / 11.30$ & $"$ & $"$ & $"$ & $?$ & $n$ & $"$ & $\begin{array}{l}\text { bedeut. Menge Cylinder, } \\
\text { einige mit Zellen. }\end{array}$ \\
\hline $28 . / 11.35$ & $"$ & $"$ & $"$ & 1,020 & $"$ & $"$ & $\begin{array}{l}\text { bedeut. Menge Cylinder, } \\
\text { einige mit Zellen. }\end{array}$ \\
\hline $3 . / 12.40$ & $"$ & " & $n$ & 1,016 & $"$ & $"$ & $\begin{array}{l}\text { bedeut. Menge Cylinder, } \\
\text { einige mit Zellen. }\end{array}$ \\
\hline $9 . / 12.45$ & $n$ & $n$ & $n$ & 1,013 & $n$ & $n$ & $\begin{array}{l}\text { bedeut. Menge Cylinder, } \\
\text { einige mit Zellen. }\end{array}$ \\
\hline $14 . / 12.50$ & $"$ & $n$ & $"$ & 1,019 & $n$ & $"$ & $\begin{array}{l}\text { bedeut. Menge Cylinder, } \\
\text { einige mit Zellen. }\end{array}$ \\
\hline
\end{tabular}

Auf Grund des Angeführten halte ich mich für berechtigt die Ansicht auszusprechen, dass in der Mehrzahl der Fälle die $\mathrm{Hg}$-Elimination während und nach der Behandlung mit $\mathrm{Hg}$ eine grössere oder geringere Reizung auf die Nieren ausübt, die sich durch das Auftreten einer mehr oder weniger bedeutenden Cylindrurie zu erkennen gibt.

Suchen wir nun zu ermitteln, welche Momente zum Auftreten einer solchen mehr oder weniger bedeutenden Cylindrurie beitragen können, so lassen sich ein ganzer. Theil denken und auch finden.

Wahrscheinlich ist es, dass eine mehr oder weniger gute Körperconstitution auf das Auftreten der Cylindrurie von Einfluss ist, doch habe ich mehr als ein Mal eine bedeutende Cylindrurie bei Personen mit einer besonders kräftigen Körperconstitution gefunden, während ich schwächliche Personen gesehen habe, die eine Behandlung mit einer bedeutenden Menge $\mathrm{Hg}$ vertrugen, ohne dass kaum ein einziger Cylinder zu entdecken war.

Unabhängig von der Körperconstitution scheint das Alter des Patienten auf das Auftreten der Cylindrurie einen nicht so unbedeutenden Einfluss auszuüben, wenigstens scheint die folgende Tabelle dieses anzugeben. 
Kann d.Behandl. mit Quecks. Cylindrurie u. Albuminurie hervorrufen? 349

Tabelle VI.

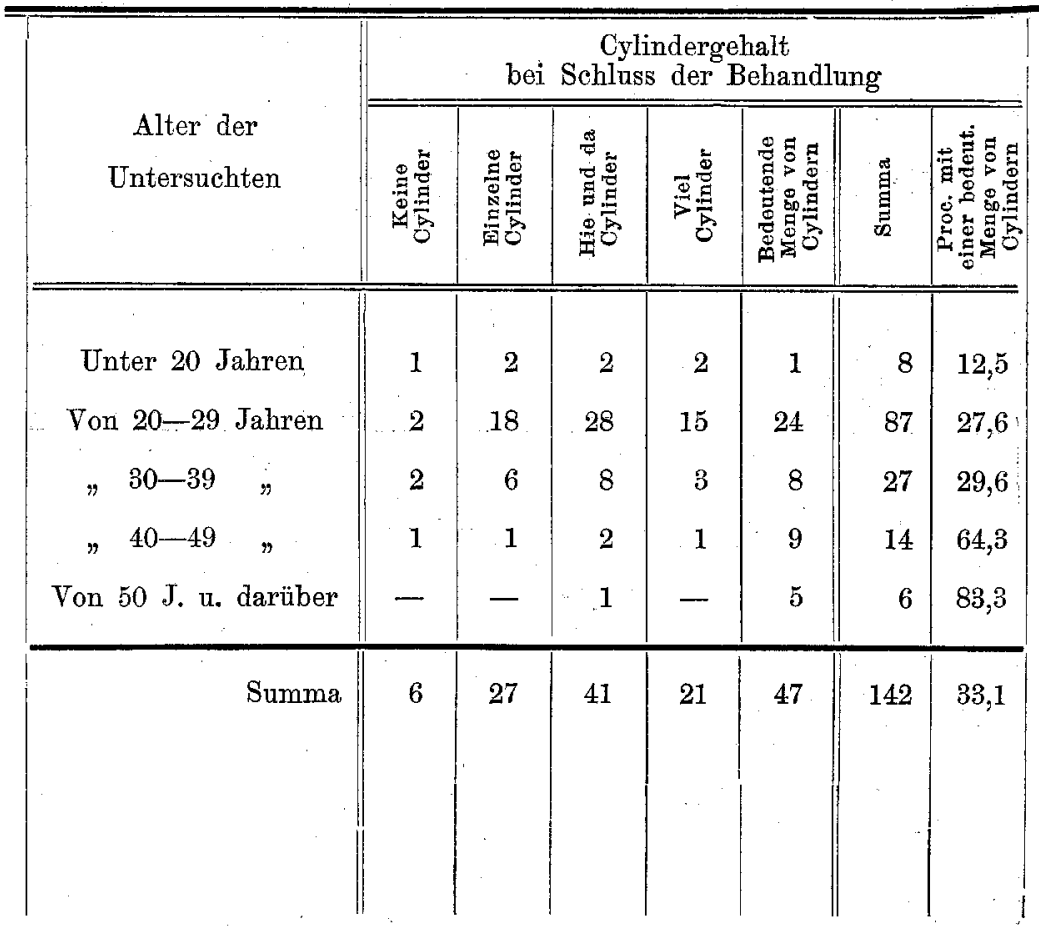

Wir sehen, wie das Procent von denen, die bei Schluss der Behandlung eine Menge Cylinder hatten, rasch mit jeder Altersclasse steigt. Der Unterschied zwischen dem Procent der Patienten unter 40 Jahren, die eine Menge Cylinder gehabt haben - 27 Procent - und dem Procent der Patienten über 40 Jahre, bei denen sich ein solcher Cylindergehalt gefunden. hat - 70 Procent - ist allzu sehr in die Augen fallend.

Einen ziemlich augenscheinlichen Einfluss auf das Auftreten der Cylindrurie scheinen auch die mehr oder weniger schweren Symptome auszuüben, wegen denen diese Patienten behandelt worden sind. Ich will hier nicht alle ihre Symptome besonders angeben, sondern ich führe sie in drei Gruppen zusammen: erster Ausbruch, Recidiv (secundäres Stadium) und Recidiv (tertiầres Stadium). Der Cylindergehalt bei Schluss der Behandlung bei den zu diesen drei resp. Gruppen gehörenden Patienten ergibt sich aus der folgenden Tabelle. 
Tabelle V.

\begin{tabular}{|c|c|c|c|c|c|c|c|}
\hline \multirow[b]{2}{*}{$\begin{array}{c}\text { Stadium } \\
\text { der Syphilis }\end{array}$} & \multicolumn{7}{|c|}{$\begin{array}{c}\text { Cylindergehalt } \\
\text { bei Schluss der Behandlung }\end{array}$} \\
\hline & 总总 & 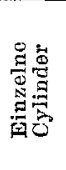 & 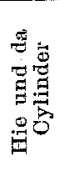 & 吾密 & 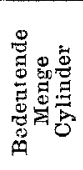 & 量 & 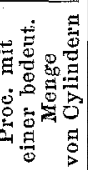 \\
\hline Erster Ausbruch & 2 & 12 & 19 & 6 & 9 & 48 & 18,7 \\
\hline Recidiviv (secund. Stad.) & 3 & 13 & 18 & 13 & 19 & 66 & 28,8 \\
\hline Recidiv (tertiär. Stad.) & 1 & - & 4 & 2 & 18 & 25 & 72,0 \\
\hline Summa & 6 & 25 & 41 & 21 & 46 & 139 & 33,1 \\
\hline
\end{tabular}

Diese verschiedenen Stadien zeigen bezüglich der Procentzahl, die eine bedeutende Cylindrurie angibt, einen höchst bemerkenswerthen Unterschied. Es will scheinen, als ob die Hg-Elimination durch die Nieren leichter eine Reizung in den Nieren bei einer Person mit tertiären Symptomen, als bei einer solchen mit frischer. Syphilis hervorrufen könnte. Es würde sich ja denken lassen, dass dieses seinen Grund zum Theil darin haben könnte, dass die tertiäre Syphilis öfter bei älteren Personen vorkommt und dass also das Alter des Patienten hierbei ein mitwirkendes Moment gewesen ist; dass aber das tertiäre Stadium an und für sich, unabhängig von dem Alter, für die $\mathrm{Hg}$-Cylindrurie predisponirt, erscheint jedoch auf Grund der folgenden beiden Tabellen als ziemlich annehmbar.

Tabelle VI.

\begin{tabular}{|c|c|c|c|c|c|c|}
\hline \multirow[b]{2}{*}{$\begin{array}{c}\text { Patienten in einem Alter } \\
\text { von mehr als } 40 \text { Jahren, } \\
\text { behandelt wegen }\end{array}$} & \multicolumn{6}{|c|}{ Cylindergehalt } \\
\hline & 吾 & 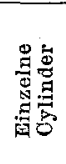 & 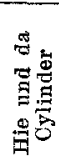 & 曾 & 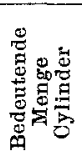 & 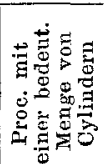 \\
\hline dem ersten Ausbruch & - & 1 & 2 & - & 3 & \\
\hline Recidiv (secund. Stad.) & - & - & 1 & - & - & \\
\hline Recidiv (tertiär. Stad.) & 1 & - & - & 1 & 10 & 83,3 \\
\hline
\end{tabular}


Tabelle VII.

\begin{tabular}{|c|c|c|c|c|c|c|}
\hline \multirow[b]{2}{*}{$\begin{array}{c}\text { Aiter der Patienten } \\
\text { dem tertiären Stadium } \\
\text { angehörend }\end{array}$} & \multicolumn{6}{|c|}{ Cylindergelit } \\
\hline & 惫总 & 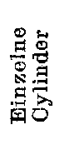 & 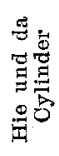 & 党苟 & 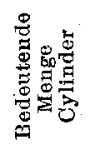 & 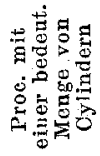 \\
\hline Unter 20 Jahren & - & - & - & - & - & - \\
\hline $20-29$ Jahre & - & 一 & 1 & - & 5 & \\
\hline $30-39 \quad "$ & - & - & 3 & 1 & 3 & 61,5 \\
\hline $40-49$ & 1 & - & - & 1 & 9 & \\
\hline 50 Jabre und darüber & 一 & - & - & 一 & 1 & 80,5 \\
\hline Summa & 1 & - & 4 & 2 & 18 & - \\
\hline
\end{tabular}

Aus Tabelle VI ersehen wir, dass von 13 Personen in einem Alter von mehr als 40 Jahren, die eine bedeutende Menge Cylinder gehabt haben, 10 dem tertiären Stadium angehörten, und finden, dass $430^{\circ}$ Proc. von denjenigen, die nicht dem tertiären Stadium angehörten, eine bedeutende Cylindrurie bekommen haben, während dieser Cylindergehalt bei 83*3 Proc. von denjenigen vorgekommen ist, die diesem Stadium angehörten. Aus Tabelle VII geht hervor, dass von 25 Personen, die dem tertiären Stadium angehörten, bei 18, beinahe ganz unabhängig vom Alter, eine bedeutende Menge Cylinder aufgetreten sind.

Einigen Einfluss scheint das Alter aber auch hier ausgeübt zu haben, indem von den Patienten, die ein Alter von weniger als 40 Jahren hatten, nur 61.5 Proc. eine bedeutende Menge Cylinder, von denjenigen dagegen, die über 40. Jahre alt waren, 83.3 Proc. eine bedeutende Cylindrurie bekommen haben.

Als eine Erklärung dafür, dass so viele von den Patienten, die dem tertiären Stadium angehörten, eine grosse Menge Cylinder bekommen haben, könnte man sich denken, dass die $\mathrm{Hg}$-Behandlung bei diesen Patienten, welche ernste Symptome gehabt haben, in der Regel kräftiger als bei der Mehrzahl der anderen Syphilitici gewesen ist und dass daher auch die $\mathrm{Hg}$ - 
Elimination sich bei ihnen grösser als bei jenen gestaltet hat. Es dürfte auf Grund dessen hier am Platze sein zu untersuchen, inwiefern eine solche Annahme Berechtigung habe, in welchem Masse eine kräftige Behandlung mit $\mathrm{Hg}$ und in welchem Masse die verschiedene Form der Finführung desselben in den Organismus für das Auftreten der Cylindrurie von Bedeutung sein könnte, und dies um so mehr, da Schwimmer in seinem Aufsatz hat andeuten wollen, dass die Thymolquecksilbereinspritzungen, die ich mitunter gegeben habe, leichter $\mathrm{zu}$ einer Reizung in den Nieren Anlass geben können, als z. B. die Einspritzungen ron Sozojodolqueeksilber, die er anwendet. In dem soeben erwähnten Aufsatz sagt Schwim mer nämlich: „Es erscheint vielleicht nicht unwesentlich, über die $\mathrm{Hg}$-Präparate sich zu orientiren, da einzelne Beobachter eben dem Gebranche dieses Medicamentes einen nachtheiligen Einfluss auf die Nierenthätigkeit zuschreiben, Andere wieder (und meine Erfahrungen sprechen auch dafür) einen günstigen. - W elander hat nach seiner mit Thymolquecksilber durchgemachten subcutanen Injectionstherapie die Steigerung der Albuminausscheidung erfahren, während man sonst diesem Medicamente keine nachtheiligen Wirkungen zuzuschreiben vermag und auch meine mit demselben gewonnenen Erfahrungen keine ungünstigen sind. Vielleicht mag die 3-4Mal wöchentlich von diesem Autor ausgefiihrte Injection zu irritirend auf den Organismus gewirkt haben."

Da Schwimmer meinen Aufsatz: „Ueber Albuminurie und Cylindrurie durch Syphilis und Quecksilber" anführt, ${ }^{1}$ ) so verwundert es mich auf's Höchste, dass er auf Grund désselben behaupten kann, dass ich Thymolquecksilberinjectionen 3-4Mal wöchentlich gebe. Wie aus meinem Aufsatze hervorgeht, gebe ich solche Injectionen höchstens jeden vierten Tag, also nicht öfter, als andere, die dieses Mittel anwenden," zu thun pflegen. In meinem Aufsatze findet sich nicht ein Wort, das Schwimmer zu seiner Behauptung berechtigt.

Wir wollen indessen nachsehen, welchen Einfluss die verschiedenen Formen der Einführung des $\mathrm{Hg}$ in den Organismus auf das Auftreten der Cylindrurie ausüben können.

1) Obschon sowohl der Titel wie die Jahreszahl unrichtig angegeben ist. 
Wenn es die Hg-Ausscheidung ist, die Anlass zum Auftreten der Cylindrurie gibt, und wenn - was wir allen Grund haben anzunelımen - das Quecksilber, unter welcher Form es auch eingegeben, auf welchen Wegen es auch in den Organismus gebracht werden mag, stets in derselben Form durch die Nieren eliminirt wird, so dürfte ja an und für sich die Form, unter der es in den Organismus gelangt, keine directe Bedeutung für die Reizung haben, die bei seiner Elimination in den Nieren erzeugt werden kann; indirect dagegen dürfte die Form, unter der das Quecksilber in den Organismus eingeführt wird, ihre Bedeutung haben können, indem bei Anwendung der einen $\mathrm{Hg}$ nicht nur in grosser Menge, sondern auch schnell absorbirt, resp. eliminirt wird, während bei Anwendung einer anderen die Menge des absorbirten, resp. eliminirten Quecksilbers wieder ganz unbedeutend sein und auch verbleiben kann. In dem einen Falle wird also eine grosse, in dem andern eine kleine Menge Quecksilber eliminirt, und da eine grosse Hg-Elimination eine bedeutendere Cylindrurie als eine kleine verursachen dürfte, so würde man in dem ersteren Falle in der Regel einen grösseren Cylindergehalt als in dem letzteren zu erwarten haben.

Die Frage gestaltet sich dann so: Wie kräftig und schnell wird das $\mathrm{Hg}$ bei Anwendung der verschiedenen Formen der Hg-Behandlung absorbirt, resp. eliminirt? (Ich nehme hier natürlicherweise nur die Hg-Präparate auf, die ich bei diesen Untersuchungen angewendet habe.)

Bei Anwendung interner Behandlung mit Hg-Pillen sind die Stärke und die Schnelligkeit der Absorption ziemlich verschieden und auch ziemalich unsicher; es gibt ja so viele Momente, die darauf einwirken können, wie z. B. die mehr oder weniger gute Beschaffenheit des Magen- und Darmcanales, die Beschaffenheit der Pillen (sind sie etwas alt und hart, was sie leicht werden, wenn man sie in einer Pappschachtel aufbewahrt, so wird von ihnen beinahe nichts absorbirt) u. s. w. Bei einer solchen inneren Behandlung ist die Hg-Elimination in der Regel nicht bedeutend, daher wir auch bei Anwendung dieser Behandlungsform keinen bedeutenden Cylindergehalt zu erwarten haben. 
Wenn Hg-Salbe in so grosser Menge auf die Hand gestrichen wird, dass die überstrichene Hautpartie mit einer dünnen Schicht bedeckt ist, so wird $\mathrm{Hg}$ in grösserer Menge absorbirt, als wenn man dieselbe Hautpartie mit Hg-Salbe e in reib t, vorausgesetzt, dass man dann nicht die Salbe, die man nicht einreiben kann, auf der Haut ausgestrichen liegen lässt. Wenn man Salbe einreibt und die eingeriebene Partie mit noch mehr Salbe überstreicht, findet eine bedeutende Absorption statt. Bei Anwendung dieser Behandlungsform sowohl wie blosser Ueberstreichungen tritt nach 25-30 Tagen stets eine bedeutende Absorption resp. Elimination von $\mathrm{Hg}$ ein. Wenden wir eine solche Behandlung an, so haben wir daher bei Schluss derselben einen bedeutenden Cylindergehalt zu erwarten.

Bei Einspritzung von Thymol-Hg und auch von Sozojodol-Hg tritt eine kräftige Absorption ein, und nach 6-7 solchen Einspritzungen ist die Hg-Elimination stets sehr bedeutend, daher wir auch bei Anwendung dieser Behandlungsformen einen grossen Cylindergehalt zu erwarten haben.

Bei Ueberstreichungen habe ich stets 6 Gr. Ung.-Hydr. $(1 \mathrm{Hg}+2 \mathrm{Fett})$ angewandt. Bei Einreibung und Ueberstreichung sind 3 Gr. Ung.-Hydr. eingerieben und 3 Gr. aufgestrichen worden. Bei Injection von Thymolquecksilber sind 1 Gr. (1 Theil essigsaures Thymolquecksilber auf 10 Theile Parafinum liquidum) eingespritzt worden. Bei Injection von Sozojodolquecksilber ist 1 Gr. (0.8 Gr. Sozojodolquecksilber und $1 \cdot 6$ Gr. Jodkalium auf 10 Theile Wasser) eingespritzt worden.

Da die innere Behandlung sich an Kraft nicht mit der Ueberstreichung und Einspritzung von $\mathrm{Hg}$ messen kann und da die Patienten, die sich in das Krankenhaus aufnehmen lassen, in der Regel einer kräftigen Behandlung bedürftig sind, so wende ich dort nur selten die Behandlung mit Pillen an, und ich habe daher auch nur einige wenige Mal Gelegenheit gehabt, den Cylindergehalt bei Personen zu untersuchen, die mit Pillen behandelt worden sind. Die folgende Tabelle zeigt, welchen Einfluss die Form der Behandlung auf das Auftreten von Cylindrurie gehabt hat. 
Tabelle VIil.

\begin{tabular}{|c|c|c|c|c|c|c|}
\hline \multirow[b]{2}{*}{$\begin{array}{c}\text { Form } \\
\text { der Behandlung }\end{array}$} & \multicolumn{6}{|c|}{ Cylindergehalt } \\
\hline & 吾 & 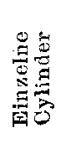 & 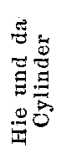 & $\frac{\Xi}{5}$ & 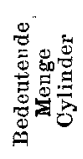 & 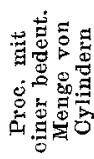 \\
\hline Pilula Hydr. & 2 & 1 & 2 & - & - & - \\
\hline Einreib. mit Ung.-Hg & - & - & 2 & - & 1 & - \\
\hline Ueberstr. mit Ung.-Hg & 1 & 12 & 14 & 6 & 28 & 45,9 \\
\hline $\begin{array}{l}\text { Einreibung u. Ueber- } \\
\text { streich. mit Ung.-Hg. }\end{array}$ & 1 & 1 & 1 & 3 & 2 & 25,0 \\
\hline Injection von Sublim. & 一 & 1. & - & - & 一 & - \\
\hline Inj. von Thymol-Hg & 1 & 9 & 9 & 4 & 8 & 25,8 \\
\hline Inject. v. Sozojodol-Hg & 1 & 3 & 13 & 8 & 8 & 24,2 \\
\hline Summa & 6 & 27 & 41 & 21 & 47 & - \\
\hline
\end{tabular}

Wir sehen, was ja zu erwarten war, dass nach der Behandlung mit Pillen nur eine unbedeutende Cylindrurie aufgetreten ist. Wir sehen ferner, dass eine bedeutende Menge Cylinder bei ungefähr 25 Proc. nach Einreibung zusammen mit Ueberstreichung und nach der Injection sowohl von Thymol-Hg wie Sozojodol-Hg aufgetreten ist und dass dieselbe Menge Cylinder sich bei 45.9 Proc. nach blosser Ueberstreichung von Hg-Salbe gezeigt hat. Zwar ist die Absorption resp. Elimination von $\mathrm{Hg}$ nach blosser Ueberstreichung sehr bedeutend, doch darf man aus obiger Tabelle nicht den Schluss ziehen, dass die Absorption und Elimination bei Anwendung dieser Behandlungsform so gross sind, dass dadurch eine grössere Reizung, als bei Anwendung einer anderen Behandlungsform auf die Nieren ausgeïbt wird. Die Ursache des grossen Procents bedeutender Cylindrurie bei Anwendung dieser Behandlungsform ist ganz sicher die, dass die Ueberstreichungen in der Regel in den schwersten Fällen, namentlich aber in einem grossen Theil der Fälle von tertïarer Syphilis angewendet werden, welches Stadium der Krankheit ja an und für sich selbst für $\mathrm{Hg}$-Cylindrurie zu prädisponiren scheint. Ausserdem sind in diesen wie auch in 
den übrigen schwereren Fällen eine viel grössere Anzahl Ueberstreichungen als in den gelinderen Fällen gegeben worden. Selbstverständlich hat die Anzahl der Ueberstreichungen einen höchst bedeutenden Finfluss auf die Grösse der Hg-Absorption resp. Elimination, und sie dürfte also denselben Einfluss auch auf den Cylindergehalt haben. Dass dieses auch der Fall ist, geht daraus hervor, dass eine bedeutende Menge Cylinder bei 57 Proc. der Patienten aufgetreten sind, die mehr als 30 Ueberstreichungen erhalten haben, während dieselbe Menge Cylinder sich nur bei $16^{\circ} 6$ Proc. von denjenigen gezeigt haben, denen weniger als 30 Ueberstreichungen gegeben worden sind.

Wären nun diese schwersten Fälle z. B. mit Sozojodol-Hg oder mit Thymol-Hg behandelt worden, in welchem Falle eine grosse Anzahl Injectionen hätte gemacht werden müssen, so würden wir ganz sicher auch bei diesen kräftigen Formen der $\mathrm{Hg}$-Behandlung ein viel grösseres Procent mit einer bedeutenden Menge Cylinder gehabt haben, als nun der Fall ist.

Wir sehen nämlich, dass auch das Sozojodolquecksilber, das nach Schwimmer keine Reizung auf die Nieren ausüben soll, in 24.2 Proc. Anlass zum Auftreten einer bedeutenden Menge Cylinder und in gleich grossem Procent zu einer nicht unbedeutenden Cylindrurie gegeben hat. Dass auch hier die Anzahl der Finspritzungen und die Dauer der Behandlung Einfluss auf den Cylindergehalt ausüben, dürfte deutlich aus folgender Tabelle hervorgehen:

Tabelle IX.

\begin{tabular}{|c|c|c|c|c|c|c|}
\hline \multirow[b]{2}{*}{$\begin{array}{c}\text { Anzahl Einspritzungen } \\
\text { von Sozojodol- } \\
\text { Quecksilber }\end{array}$} & \multicolumn{6}{|c|}{ Cylindergehalt } \\
\hline & 焉 & 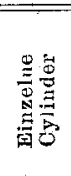 & 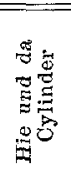 & 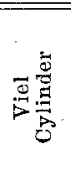 & 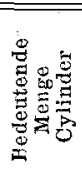 & 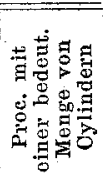 \\
\hline 7 Einspritzungen & - & - & 5 & 3 & 5 & 38,5 \\
\hline 6 & - & 1 & 2 & 3 & $3 \cdots$ & 33,3 \\
\hline 5 & 1 & 2 & 6 & 2 & $\rightarrow$ & 0,0 \\
\hline
\end{tabular}


Kann d. Behandl. mit Quecks. Cylindrurie u. Albuminurie hervorrufen? 357

Wir finden nach 5 Einspritzungen bei 0 Proc., nach 6 Einspritzungen bei 33.3 Proc. und nach 7 Einspritzungen bei $38 \cdot 5$ Proc. eine bedeutende Menge Cylinder.

Die Form, unter welcher das Quecksilber in den Organismus eingeführt wird, hat an und für sich selbst ganz sicher nur eine sehr geringe Bedeutung; von Gewicht ist die Form nur insofern, als bei Anwendung der einen $\mathrm{Hg}$ in grösserer Menge als bei Anwendung der anderen absorbirt, resp. eliminirt wird. Die hauptsächlichste Bedeutung hat die Menge absorbirtes, resp. eliminirtes $\mathrm{Hg}$; bei Anwendung derselben Behandlungsform ist es die Dauer der Behandlung, die in dieser Hinsicht Bedeutung hat.

Wir haben zwar gesehen, dass die Cylindrurie in der Regel ziemlich bald verschwindet und dass die Reizung, welche die $\mathrm{Hg}$-Elimination verursacht, übergehend ist, aber kann sie die Widerstandskraft der Nieren nicht insofern verringern, dass bei einer folgenden Behandlung eine Cylindrurie leichter entsteht? Ich habe in der folgenden Tabelle $\mathrm{X}$ den Cylindergehalt bei Patienten zusammengestellt, die ein, zwei, drei oder mehrere Mal behandelt worden sind.

Tabelle $\mathbf{X}$.

\begin{tabular}{|c|c|c|c|c|c|c|}
\hline \multirow[b]{2}{*}{$\begin{array}{c}\text { Mit Hg } \\
\text { behandelt }\end{array}$} & \multicolumn{6}{|c|}{ Cylindergehalt } \\
\hline & 蒡 & 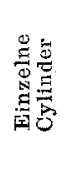 & 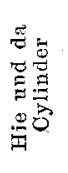 & $\frac{\sqrt[5]{0}}{8}$ & 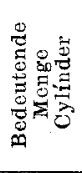 & 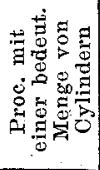 \\
\hline das erste Mal & 3 & 18 & 26 & 11 & 19 & 27,7 \\
\hline$n$ zweite $"$ & 2 & 6 & 7 & 6 & 9 & 30,0 \\
\hline$"$ dritte $"$ & - & 3 & 2 & - & 5 & 50,0 \\
\hline mehr als drei Mal & 1 & - & 6 & 4 & 14 & 56,0 \\
\hline Summa & 6 & 27 & 41 & 21 & 47 & - \\
\hline
\end{tabular}

Wir finden, dass das Procent der Patienten, die eine Menge Cylinder im Harn gehabt haben, mit der Anzahl der 
von ihnen durchgemachten $\mathrm{Hg}$-Behandlungen wächst, was ja dafür sprechen würde, dass eine frühere $\mathrm{Hg}$-Behandlung für das Auftreten einer bedeutenden Cylindrurie bei einer späteren prädisponirt. Aber hierbei ist nicht ausser Acht zu lassen, dass die meisten der Patienten, bei denen bei der dritten oder einer späteren Hg-Behandlung eine bedeutende Menge Cylinder im Harn aufgetreten sind, sich im tertiären Stadium befunden haben und dass ihre Behandlung deshalb kräftig und langdauernd gewesen ist, daher diese Tabelle keinen Beweis für die Richtigkeit eines Schlusses in der von mir hier oben angedeuteten Richtung geben kann. Hierzu will ich noch fügen, dass ich mehr als einmal bei Patienten ein wenig mehr Cylinder bei Schluss ihrer ersten als bei Schluss ihrer zweiten HgBehandlung gesehen habe, trotzdem diese ganz ebenso stark wie die erste sein konnte. Die Regel ist jedoch die, dass ceteris paribus bei einer folgenden $\mathrm{Hg}$-Behandlung Cylindrurie in keinem geringeren Grade als bei der vorhergegangenen auftritt.

Man hat gesagt, dass die Einnahme von Jodkalium die Quecksilberelimination vermehre. Sollte dieses der Fall sein, so mïsste man ja bei Einnahme von Jodkalium während oder gleich nach einer $\mathrm{Hg}$-Behandlung eine Vermehrung des Cylindergehaltes erwarten können. Nun habe ich keine vermehrte Elimination von $\mathrm{Hg}$ bei Einnahme von Jodkalium zu constatiren vermocht. (Die von mir angewendete Untersuchungsmethode ist, wie Schillberg gezeigt hat, für die $\mathrm{Hg}$-Untersuchung nicht ganz zweckmässig, sobald sich auch Jodkalium findet.) In einem Theil Fälle, wo Jodkalium und $\mathrm{Hg}$ gleichzeitig mit der Hg-Behandlung oder nach Abschluss derselben gegeben worden sind, habe ich indessen keine Vermehrung der Cylindrurie als Folge davon constatiren können.

Wir haben nun mehrere Momente betrachtet, die für das Auftreten einer grösseren oder geringeren Menge Cylinder ohne Zweifel Bedeutung haben können, doch gibt es aber auch Fälle, für welche eine derartige Erklärung durchaus nicht anwendbar ist. So kann es zuweilen geschehen, dass ein junger und kräftiger Patient, der wegen gelinden Symptomen zum ersten Mal behandelt wird, schon nach 10-15 Tagen eine bedeutende Cylindrurie haben kann, während ein anderer, älterer Patient im 
tertiären Stadium, der mehrere $\mathrm{Hg}$-Behandlungen durchgemacht hat, z. B. bei einer so lange dauernden und kräftigen $\mathrm{Hg}-\mathrm{Be}-$ handlung wie 40 Ueberstreichungen nicht einen einzigen Cylinder in seinen Harn bekommt. Derartige Fälle können wir in keiner anderen Weise als durch die Annahme erklären, dass es für die Quecksilbercylindrurie, gleichwie für die Quecksilberstomatitis, eine verschiedene individuelle Disposition gibt. Im Grossen und Ganzen findet man auch, dass das Auftreten einer mehr oder weniger bedeutenden Cylindrurie hauptsächlich in individueller Disposition seinen Grund hat, obschon die von mir angegebenen Momente dazu beitragen können.

In meinem vorigen Aufsatz habe ich die Frage berührt, inwiefern die individuelle Disposition für die Cylindrurie mit derjenigen für die Stomatitis zusammenfällt, und sie dahin beantwortet, dass man wohl bei einem Patienten mit schwerer Stomatitis nicht selten auch eine recht bedeutende Cylindrurie finden kann, dass dieses aber bei weitem nicht immer der Fall ist; man findet Personen mit bedeutender Stomatitis ohne $\mathrm{Cy}-$ lindrurie, gleichwie man Personen mit bedeutender Cylindrurie findet, bei denen das Zahnfleisch völlig gesund ist. Meine letzten Untersuchungen haben die Richtigkeit dieser meiner Ansicht vollständig bestätigt. So bekam z. B. A. nach 11 Ueberstreichungen eine höchst bedeutende Stomatitis, ohne dass sich in ihrem Harn ein einziger Cylinder entdecken liess. Ganz entgegengesetzt war das Verhältniss bei J., wo sich nach 30 Ueberstreichungen im Harn Spuren von Albumin mit einer bedeutenden Menge Cylinder fanden, das Zahnfleisch aber völlig gesund war. Eine bedeutende Cylindrurie sieht man nicht so selten mit lockerem Zahnfleisch vereinigt; auf der anderen Seite kann man aber absolut gesundes Zahnfleisch und den Harn völlig frei von Cylindern finden, ungeachtet eine äusserst kräftige Behandlung angewendet worden ist; dieses traf z. B. in Fall IX ein. Man würde hier anmerken können, dass das gesunde Zahnfleisch - wie F in g e r herrorhebt - anzeigt, dass die Behandlung nicht richtig ausgeführt worden ist, und dass man hierin die Ursache zu sehen hat, dass z. B. in dem genannten Falle weder eine Andeutung von einer Stomatitis, noch von einer Cylindrurie zu finden gewesen ist; eine bei Schluss der 
Behandlung ausgeführte Untersuchung des Harns auf Quecksilber hat hier aber eine bedeutende Menge $\mathrm{Hg}$ ergeben. Dieser und eine Menge andere derartige Fälle geben mir das Recht, fortfahrend an der Ansicht festzuhalten, die ich in meinem vorigen Aufsatze ausgesprochen habe. Da es seine praktische Bedentung hat, will ich diese Ansicht hier anführen: „Der Urin ohne Eiweiss und ohne Cylinder berechtigt ebensowenig wie völlig gesundes Zahnfleisch zu dem Schlusse, dass nur eine unbedeutende Menge $\mathrm{Hg}$ absorbirt worden ist; die Kenntniss von der Grösse der absorbirten Hg-Menge wird nur durch Untersuchung des Urins oder der Foeces auf $\mathrm{Hg}$ erhalten."

Man würde ja anmerken können, dass die $\mathrm{Hg}$-Elimination diese Wirkung zwar auf Patienten ausübt, die an Syphilis leiden, aber eine ganz andere Wirkung auf Patienten hat, die diese Krankheit nicht haben. Um nun diese Frage zu beantworten, kam ich mit zwei Personen, die nie an Syphilis gelitten hatten, überein, ihnen $\mathrm{Hg}$-Behandlung zu geben, der einen Einspritzungen von Thymol-Hg, der anderen Ueberstreichungen mit Ung.-Hg. Die erstere bekam nach den Einspritzungen so bedeutende Infiltrate, dass ich mich genöthigt sah, mit ihnen aufzuhören und anstatt ihrer Ueberstreichungen zu geben; nach 2 Einspritzungen und 6 Ueberstreichungen stellte sich eine so intensive Stomatitis ein, dass ich die $\mathrm{Hg}$-Behandlung nicht länger fortsetzen konnte; bei Beginn der Behandlung enthielt der Harn keine Cylinder und bei Schluss derselben nur einzelne (also eine bedeutende individuelle Disposition für mercurielle Stomatitis, aber nicht für mercurielle Cylindrurie). Bei der anderen Person zeigten sich nach 30 Ueberstreichungen Cylinder nur hie und da. Es trat also in beiden Fällen nur eine ganz unbedeutende Cylindrurie ein. Die Fälle sind $z u$ wenige, um in irgend einer Weise zu dem Schlusse zu berechtigen, dass Cvlindrurie nicht bei Personen auftritt, die nie Syphilis gehabt haben. Das Gegentheil scheint aus einem andern von mir beobachteten Fall hervorzugehen, wo das $\mathrm{Hg}$ eine höchst bedeutende Cylindrurie und auch Albuminurie hervorrief. In diesem Falle hatte der Patient auf Grund verdächtiger syphilitischer Symptome eine Hg-Behandlung durchgemacht, der Verlauf der Krankheit liess es aber als höchst wahrscheinlich erscheinen, 
dass er keine Syphilis hatte. Was die Albuminurie anbelangt, so will ich später zwei Fälle anführen, wo Albuminurie bei nicht syphilitischen Personen nach Anwendung von Quecksilber aufgetreten ist. Dieses spricht ja bestimmt dafür, dass das Quecksilber - gleichviel ob Syphilis vorliegt oder nicht eine Reizung in den Nieren verursachen kann.

Kann nun eine Behandlung mit Quecksilber auch zum Entstehen von Albuminurie Anlass geben? Wenn dieses der Fall ist, findet sich dann etwas Eigenthümliches im Auftreten dieser Albuminurie? Findet sich ein Zusammenhang zwischen dem Auftreten der $\mathrm{Hg}$-Cylindrurie und der $\mathrm{Hg}$-Albuminurie?

Gleichwie bei der Hg-Cylindrurie müssen wir auch bei der $\mathrm{Hg}$-Albuminurie die Bedingung aufstellen, dass sie im Laufe der Behandlung auftritt, ${ }^{1}$ ) und dass sie nicht nur bis zum Schluss derselben dauert, sondern wenigstens noch einige Zeit darïber hinaus anhält, ehe man das Recht hat zu behaupten, dass sie durch die Elimination des Quecksilbers durch die Nieren hervorgerufen worden ist. Die Erfüllung der beiden ersten Forderungen ist leicht zu constatiren gewesen; schwerer hat dieses dagegen, aus ganz demselben Grunde wie bei der Cylindrurie, bei der letzten gehalten, indem nämlich die $\mathrm{Pa}$ tienten in der Regel gleich nach Schluss der Behandlung das Krankenhaus verlassen haben; aber wenn ich auch nicht in der Lage gewesen bin, die Patienten nach Schluss der Behandlung ununterbrochen zu beobachten, so hat mir doch in mehr als einem Fall die Erfahrung gezeigt, dass einige Zeit nach Schluss der Behandlung, wo die $\mathrm{Hg}$-Elimination also bedeutend vermindert war, die Albuminurie verschwunden gewesen ist, was ja in einem höchst bedeutenden Grade dafür spricht, dass diese Albuminurie wirklich in der Hg-Elimination ihre Ursache gehabt hat.

Zur Untersuchung auf Albumin habe ich sowohl Salpetersäure, wie 20procentige Trichloressigsäure angewendet. Oft ist es geschehen, dass dies letztgenannte Mittel eine deutliche Eiweissreaction gegeben hat, wo Eiweiss mit Salpetersäure nicht zu entdecken war. In mehreren Fällen ist der Albumingehalt

1) In ein paar Fällen, wo Thymolquecksilber eingespritzt worden ist, habe ich die Albuminurie erst einige Tage nach der letzten Einspritzung 
so gering gewesen, dass er sich nur mit Trichloressigsäure hat entdecken lassen, in anderen Fällen wieder so gross, dass auch Salpetersäure Reaction auf Eiweiss gegeben hat.

In 10 Fällen ist der Albumingehalt nur mittelst der Trichloressigsäure zu constatiren gewesen. In 7 von diesen Fällen sind Ueberstreichungen mit Ung.-Hydr., in 2 Einspritzungen von Thymolquecksilber und in 1 Einspritzungen von Sozojodolquecksilber gegeben worden. In sämmtlichen diesen Fällen ist bei Beginn der Behandlung keine Albuminurie vorhanden gewesen, sondern sie ist erst zwischen dem 18. und 30. Tage der Behandlung aufgetreten. Nachdem die Albuminurie sich erst einmal eingestellt gehabt hatte, ist sie während der ganzen Zeit zu beobachten gewesen; welche die Behandlung noch dauerte. In drei Fällen habe ich einige Wochen nach Schluss der Behandlong Gelegenheit gehabt, den Harn der Patienten zu untersuchen, und ich habe dann keine Spur von Albumin darin entdecken können.

In 8 Fällen hat sich Eiweiss nicht nur mit Trichloressigsäure, sondern auch mit Salpetersäure nachweisen lassen. Zuerst konnte es mit der Trichloressigsäure, und erst ein paar oder einige Tage später auch mit der Salpetersäure nachgewiesen werden. In dreien von diesen 8 Fällen habe ich einige Wochen nach Schluss der Behandlung den Harn der Patienten untersuchen können, und es hat sich dann gezeigt, dass zuerst die Albuminreaction der Salpetersäure und einige Zeit nachher erst die der Trichloressigsäure verschwunden war. In einem Falle hat sich eine mit der Trichloressigsäure nachweisbare Albuminmenge noch 5 Wochen und in einem anderen noch $2 \%$ Monate nach Schluss der Hg-Behandlung gezeigt.

Der Verlauf der Albuminurie ist ja sehr eigenthümlich, und auf Grund der Uebereinstimmung, die sich zwischen der Menge des Albumins und derjenigen des eliminirten $\mathrm{Hg}$ findet, spricht er deutlich für einen Causalzusammenhang zwischen dieser Albuminurie und der $\mathrm{Hg}$-Elimination.

auftreten sehen, was sich ja auf Grund der Absorption bei dieser Behandlungsform leicht erklären lässt. Diese Fälle gehören indessen nicht der Untersuchungsserie an, die ich hier bespreche. 
Aber diese Albuminurie ist ausserdem durch eine höchst bedeutende Cylindrurie gekennzeichnet. Die Albuminurie ist in allen 18 Fällen unbedeutend gewesen, während die Cylindrurie sich in 15 von ihnen als höchst bedeutend und in den drei übrigen als bedeutend erwiesen hat. Ich habe nicht so wenig Fälle von Albuminurie aus anderen Grïnden untersucht, und ich habe in ihnen beinahe stets eine gewisse Uebereinstimmung zwischen der Menge des Eiweisses und der Anzahl der Cylinder gefunden; bei der Mercurialalbuminurie ist dieses Verhältniss ein entgegengesetztes. Man könnte sich darüber verwundern, eine so bedentende Menge Cylinder zu finden, wenn die Albuminurie kaum nachweisbar ist, aber wenn man solche Fälle verfolgt, findet sich die Erklärung bald. Je nachdem die Behandlung fortschreitet, treten nämlich immer mehr Cylinder auf, bis ihre Anzahl in der Regel bedeutend ist; und erst jetzt tritt die Albuminurie ein. Von den 18 Fällen, deren ich oben Erwähnung gethan habe, ist in 12 bei Beginn der Behandlung nicht ein einziger Cylinder zu entdecken gewesen, in 3 anderen liessen sich ein paar Cylinder nachweisen und in den übrigen 3 Fällen ist bei Beginn der Behandlung keine Untersuchung auf Cylinder ausgeführt worden (aber auch in diesen Fällen vermehrte sich der Cylindergehalt im Laufe der Behandlung); wie ich oben erwähnt habe, war der Cylindergehalt in sämmtlichen 18 Fällen beim Eintritt der Albuminurie bedeutend.

Aber man findet eine Veränderung nicht nur in der Anzahl der Cylinder, sondern auch in ihrer Beschaffenheit. Im Anfange sind die Cylinder nur hyalin und mitunter ziemlich sehwer zu entdecken; oft treten unter diesen hyalinen Cylindern später körnige auf; schliesslich kann man auf den Cylindern hie und da die eine oder andere Zelle unterscheiden, und mehr als einmal lassen sich zuletzt mehr oder weniger vollständige Epithelcylinder sehen. Finden sich diese Cylinder, so ist stets Albuminurie vorhanden; dagegen können Cylinder mit der einen oder anderen Zelle auftreten, ohne dass Albuminurie sich zu finden braucht, gleichwie es geschehen ist, dass sich Albumin zeigte, ohne dass andere als hyaline Cylinder zu entdecken waren. Wenn die Cylindrurie nach Schluss der Behandlung rerschwindet, so verschwinden zuerst diese Cylinder mit 
Zellen, und sodann vermindert sich allmälig auch die Anzahl der feinkörnigen und hyalinen Cylinder, die aber erst eine längere oder kürzere Zeit nach dem Auf́hören der Albuminurie ganz verschwunden sind, was ich sowohl bei der jetzigen wie bei früheren Untersuchungsserien bei Patienten controliren konnte, die nach einiger Zeit wegen einem Recidiv der Syphilis wieder in das Krankenhaus aufgenommen wurden.

Wir finden also eine ununterbrochene Steigerung des Cylindergehaltes, oft eine Veränderung der Beschaffenheit der Cylinder, das Auftreten von Albuminurie, die sich zuerst nur mittelst eines besonders empfindlichen Reagens, später aber mittelst Salpetersäure nachweisen lässt, und wir finden dieses alles im Laufe der Behandlung oder in der nächsten Zeit nach Abschluss derselben, d. h. je nach dem die Hg-Elimination steigt; wenn diese anfängt sich zu vermindern, so vermindert sich auch die Albuminurie; wenn diese verschwunden ist, finden sich zwar noch Cylinder, aber ihre Beschaffenheit und Anzahl verändert sich mehr und mehr, bis auch sie schliesslich verschwinden, wenn die Elimination von $\mathrm{Hg}$ höchst bedeutend vermindert ist. (Als ein Beispiel will ich auf den weiter vorn angeführten Fall $\mathrm{X}$ hinweisen.)

Es kann bei der Beurtheilung dieser Cylindrurie und Albuminurie wohl nicht gern der geringste Zweifel herrschen, sondern wir müssen sagen: sie steht im Causalzusammenhang mit der Hg-Elimination - es ist eine mercurielle Cylindrurie, eine mercurielle Albuminurie. ${ }^{1}$ )

Aber es gibt Albuminurie bei Syphiliskranken, die schwerer zu erklären ist, z. B. wenn man schon bei Beginn der. Be-

1) $\mathrm{Ob}$ nun das Eiweiss Nucleoalbumin oder Serumalbumin gewesen ist, vermag ich nicht zu entscheiden; dass mitunter Nucleoalbumin vorgekommen ist, kann ich auf Grund der kleinen wolkenförmigen hellen Trübung, die sich ein Stück über dem scharfen Albuminringe bildete, mit Sicherheit behaupten. Ich will auch hervorheben, dass ich sehr oft eine höchst bedeutende Menge Cylindroiden sowohl beim Auftreten einer Menge Cylinder, wie beim Vorkommen von Albuminurie gefunden habe.

Aber wenn auch das Albumin Nucleoalbumin gewesen und die Bedeutung dieses Albumins geringer als die des Serumalbumins ist, so gibt sein Vorkommen doch eine Reizung in den Nieren an, da es stets mit dein'Auftreten einer bedeutenden Cylindrurie verbunden gewesen ist. 
handlung Albuminurie findet. In der Literatur findet sich mehr als ein solcher Fall als eine durch Syphilis hervorgerufene Albuminurie beschrieben, wozu sich doch ganz sicher viele Male keine Berechtigung gefunden hat.

Verschwindet eine solche Albuminurie im Laufe der Behandlung, so ist és ja möglich, aber nicht bewiesen, dass sie in der Syphilis ihren Grund gehabt hat; verschwindet sie nicht, so ist dieses nicht als ein Beweis dafür aufzufassen; dass sie mit der Syphilis nicht in Zusammenhang gestanden hat. (Hierüber, wie auch in Betreff der Bedingungen, um eine Albuminurie möglicherweise als Albuminuria syphilitica auffassen zu können, siehe meinen Aufsatz über Cylindrurie und Albuminurie S. 4-7.) Wie ich in diesem Aufsatz hervorgehoben habe, muss man sich mehr als einmal bedenken und genau eine Menge Verhältnisse prüfen, ehe man das Urtheil fällen darf, dass es die syphilitische Krankheit an und für sich ist, in der man die Ursache der Albuminurie zu sehen hat.

Ich habe nun 8 Fälle beobachtet, wo die Patienten bei ihrer Aufnahme in das Krankenhaus Albuminurie hatten, die ich nicht auf Rechnung der Syphilis schreiben konnte; dieselbe hat sich die ganze Zeit über gefunden, welche die Patienten im Krankenhause zubrachten, und sich nicht im geringsten durch die Hg-Behandlung beeinflusst gezeigt.

Fall XII. P. ist schon zweimaI voraus im Krankenhause gepflegt worden und hat dann die ganze Zeit über eine unbedeutende Albuminurie gehabt. Er wurde jetzt zum dritten Mal am 17./4. wegen Roseola und Pap. aufgenommen; auch dieses Mal hatte er während der ganzen Zeit seiner Behandlung eine unbedentende Albuminurie.

Fall XIII. J. wurde in das Krankenhaus am 17.4. wegen Sclerose an der Pars pendula aufgenommen; da er keine Anschwellung der Drüsen hatte, wurde die Sclerose excidirt; er litt an Alcoholismus chronicus; im Harn fanden sich Spuren von Albumin. Am 2./5. begannen Roseola aufzutreten. Er wurde am 1.6. aus dem Krankenhause entlassen, nachdem er 30 Ueberstreichungen erhalten hatte. Er hatte während der ganzen Zeit seines Aufentbaltes im Krankenhause eine unbedeutende Albuminurie.

Fall XIV: K. wurde in das Krankenhaus am 21./4. wegen papulopustulösem Syphilid aufgenommen; er hatte einen Herzfehler und es fand sich gelinde Albuminurie, die sich die ganze Zeit über erhielt, bis er am 19.6. aus dem Krankenhause entlassen wurde. Er hatte 7 Thymol-Hg-Einspritzungen erhalten. 
Fall XV. P. wurde in das Krankenhaus zum ersten Mal am 24./5. wegen Sclerose aufgenommen. Am 20./6. traten Roseola auf; er bekam von diesem Tage bis zum 15.77. 6 Einspritzungen von Sozojodol-Hg; hatte während der ganzen Zeit seines Aufenthaltes im Krankenhause gelinde Albuminurie.

Fall XVI. Dieselbe Person wie im vorigen Fall wurde in das Krankenhaus am 5./9. wegen Papulae mucosae anfgenommen. Sie wurde aus dem Krankenhause nach 35 Ueberstreichungen am 9./10. entlassen; hatte die ganze Zeit über gelinde Albuminurie.

Fall XVII. W. wurde in das Krankenhaus am 7./6. wegen Papulae mucosae aufgenommen. Er wurde aus dem Krankenhause am 8.7. nach 30 Ueberstreichungen entlassen; Albuminurie die ganze Zeit über in unbedeutendem Grade.

Fall XVIII. C. wurde in das Krankenhaus am 22./8. wegen Sclerose und Roseola aufgenommen; bekam bis zum 18./9. 6 Sozojodol-Hg-Einspritzungen; die ganze Zeit über gelinde Albuminurie.

Fall XIX. C. wurde in das Krankenhaus wegen Sclerose und papulösem Syphilid aufgenommen; bekam 30 Ueberstreichungen; hatte die ganze Zeit über, wo er sich im Krankenhause aufhielt, unbedeutende Albuminurie.

Nicht in einem dieser Fälle betrachte ich mich für berechtigt zu behaupten, dass die Albuminurie durch die Syphilis hervorgerufen gewesen ist. In einem Falle war wahrscheinlich Alcoholismus chronicus im Verein mit einem Fettherzen, in einem anderen ein organischer Herzfehler (der Patient starb nach einigen Monaten daran) die Ursache der Albuminurie. Die Ursache der Albuminurie in den übrigen Fällen habe ich nicht finden können, ebensowenig wie es mir mitunter bei Personen, die nicht an Syphilis litten, möglich gewesen ist zu erklären, weshalb sie in ihrem Harn Spuren von Albumin hatten.

Ausser diesen 8 Fällen habe ich 3 andere beobachtet, wo die Patienten bei ibrer Aufnahme in das Krankenhaus ansgebreitete gummöse Geschwüre and dann auch gelinde Albuminurie hatten; in zweien dieser Fälle fanden sich ein paar hyaline Cylinder, im dritten aber liessen sich keine entdecken. Dieses kann ja zum Theil gegen die Annahme sprechen, dass die Syphilis die Ursache der Albuminurie war, um so mehr, da die Albuminurie während der ganzen Zeit der Behandlung anhielt, ungeachtet die gummösen Geschwüre schnell heilten.

Nur einen Fall habe ich Gelegenheit gehabt zu beobachten, wo die Albuminurie im Laufe der Behandlung versehwunden ist, und dieser Fall würde sich möglicherweise als ein Fall von Albuminuria syphilitica deuten lassen. 
Fall XX. D., 16 Jahre alt, wurde am 29./8. wegen dem ersten Ausbruch der Syphilis in das Krankenhaus aufgenommen; er hatte da Albuminurie (Menge des Albumins 0,03 Proc.), sowie Cylinder hie und da; bekam eine Einspritzung von Sozojodolquecksilber.

3./9. hatte er 0.06 Proc. Albumin, sowie einzelne Cylinder; 2. Einspritzung von Sozojodol-Hg.

8./9. hatte er unbedeutende Spuren von Albumin und hier und da Cylinder; 3. Einspritzung von Sozojodol-Hg.

Seit diesem Tage liess sich kein Albumin mehr entdecken. Den 30./9. war der Patient frei von Symptomen, nachdem er 7 Einspritzungen von Sozojodol-Hg. erhalten hatte; der Harn war jetzt frei von Albumin; es fanden sich in ihm viele Cylinder.

Der Patient erwähnte bei seiner Aufnahme in das Krankenhaus, dass er 2 Jahre vorher Scarlatina gehabt hatte; ob sein Harn damals oder später eiweisshaltig gewesen war, wusste er nicht. Bei seiner Aufnahme in das Krankenhaus vermuthete ich indessen, dass seine Albuminurie eine Folge des von ihm durchgemachten Scharlachfiebers war. Dieses scheint jedoch nicht der Fall gewesen za sein. Da seine Albuminurie im Laufe der Behandlung gleichzeitig mit den syphilitischen Symptomen verschwand, so liegt es ja innerhalb der Grenzen der Möglichkeit, dass hier wirklich eine Albuminuria syphilitica vorgelegen hat.

Schwimmer sagt, dass in seinen Fällen Albuminurie zwar im Laufe der Behandlung entstanden, dass sie aber auch wieder im Laufe der Behandlung verschwunden sei. In den Jahren, wo ich regelmässig Untersuchungen auf Eiweiss bei allen Patienten ausgeführt habe, die der Behandlung mit $\mathrm{Hg}$ unterworfen wurden, habe ich nur in einzelnen Fällen eine unbedeutende Albuminirie für einen oder ein paar Tage im Laufe der Behandlung auftreten sehen. In der Zeit, wo ich diese letzte Serie von Untersuchungen ausgeführt habe, bin ich nur $4 \mathrm{Mal}$ in der Lage gewesen, solche Fälle zu beobachten.

So geschah es z. B., dass ein Patient, der zum ersten Male im Krankenhause gepflegt wurde irn Laufe der Behandlung für einen Tag. Albuminurie erhielt; auch jetzt, wo er wegen einem Recidiv der Syphilis behandelt wurde, bekam er einige Tage nach seiner Aufnahme in das. Krankenhaus Albuminurie, die 6 Tage andanerte. Als er 13 Ueberstreichungen erhalten hatte, liessen sich in seinem Harn minimale Spuren. von Albumin mittelst Trichloressigsäure entdecken; nach diesem Tage zeigte sich keine Albuminurie mehr; er wurde aus dem Krankenhause entlassen, als er 30 Ueberstreichungen erhalten hatte.

Was die Ursache der Albuminurie in diesem wie in den drei anderen gleichartigen Fällen gewesen ist, vermag ich nicht. zu entscheiden; von der Hg-Behandlung können wir ja ganz 
sicher absehen. In gleichartigen Fällen, die Schwimmer beobachtet hat, sieht er die Ursache der Albuminurie in der Syphilis, doch bezweifle ich, dass er darin Recht hat, zumal kein beweisender Grund für diese Ansicht dargelegt worden ist. Ich für meinen Theil habe zufälligerweise ein paar Mal bei nicht syphilitischen Personen eine solche Albuminurie ganz plötzlich in unerklärlicher Weise entstehen und ebenso plötzlich wieder verschwinden sehen; es erscheint mir nicht als unmöglich, dass dieses, da es bei nicht syphilitischen Personen geschehen kann, auch bei syphilitischen einzutreffen vermag, ohne dass wir deshalb das Recht haben, die Schuld dafür auf die Syphilis zu schieben.

Dass Quecksilber Albuminurie auch bei nicht syphilitischen Personen hervorrufen kann, habe ich in meinem rorigen Aufsatz gezeigt, wo sich erwähnt findet, dass eine Krankenpflegerin, die während einer langen Zeit Hg-Einreibungen gegeben hatte, Symptome von $\mathrm{Hg}$-Vergiftung mit Albuminurie bekam, welches alles aber wieder verschwand, nachdem sie einige Zeit aufgehört hatte, solche Einreibungen auszuführen. Der zweite Fall war ein Mann, der, um sich von Ungeziefer zu befreien, seinen Körper und seine Unterkleider mit einer Menge Mercurialsalbe und metallischem Quecksilber eingeschmiert hatte und dann vierzehn Tage gegangen war, ohne seine Unterkleider zu wechseln; er bekam eine höchst bedeutende Stomatitis und auch Albuminurie; sein Harn enthielt eine bedeutende Menge Hg. Nach einiger Zeit war sowohl die Stomatitis, wie die Albuminurie verschwunden; auch die Hg-Elimination durch die Nieren war bedeutend vermindert.

Die Frage, inwiefern die Hg-Elimination durch die Nieren eine Reizung in den Nieren hervorruft, glaube ich auf Grund der obigen Untersuchungen berechtigt $z u$ sein dahin zu beantworten, dass in der Mehrzahl der Fälle eine solche Reizung ịn einem grösseren oder geringerem Grade entsteht. Frägt man wieder, ob diese Reizung von der Bedeutung ist, dass sie für den Augenblick oder für die Zukunft einen Schaden verursachen kann, so glaube ich die Antwort geben zu können, dass dieses wenigstens in der Regel nicht der Fall ist. Dieses hindert jedoch nicht, dass es in einzelnen Fällen eintreffen 
kann, z. B. in dem Falle, wo Albuminurie und bedeutende Cylindrurie sich noch 10 Wochen nach beendigter Hg-Behandlung fanden. Es scheint mir daher ebenso nothwendig zu sein, während einer kräftigen $\mathrm{Hg}$-Behandlung den Harn zu controliren (ihn wenigstens mittelst eines empfindlichen Reagens auf Albumin zu untersuchen), wie die Haut, das Zahnfleisch und den Darmcanal einer Controle zu unterwerfen, denn das Auftreten von Albuminurie zusammen mit einer bedeutenden Cylindrurie, besonders wenn sich Epithelcylinder zeigen, ist ein recht sicheres Zeichen, dass man Acht geben und mit der ferneren Verabreichung von Quecksilber vorsichtig sein muss, bis die Reizung in den Nieren sich vermindert hat oder verschwunden ist.

Dieses hat seine Giltigkeit für Personen, die bei Beginn der Behandlung nicht die geringste Reizung in den Nieren haben. Wie wirkt nun die $\mathrm{Hg}$-Elimination auf Personen ein, die man einer kräftigen $\mathrm{Hg}$-Behandlung unterwerfen muss und die bei Beginn dieser Behandlung bereits Albuminurie haben?

In den Fällen XII-XIX und in den drei Fällen, wo ausgebreitete gummöse Geschwüre auftraten, fand sich bei der Aufnahme der Patienten in das Krankenhaus eine gelinde Albuminurie, die während der ganzen Behandlung anhielt, ohne dass ich eine nachweisbare Vermehrung derselben za beobachten vermochte. Dahingegen fand ich in der Nehrzahl dieser Fälle eine bedeutende Veränderung des Cylindergehaltes, die ganz sicher auf Rechnung der Hg-Elimination zu schreiben ist. Von 11 Personen hatten 7 bei ihrer Aufnahme in das Krankenhaus keine Cylinder und 4 einzelne Cylinder; bei Schluss der Behandlung. fanden sich einzelne Cylinder bei 1, Cylinder hie nnd da bei 2, viel Cylinder bei 2 und eine bedeutende Menge von Cylindern bei 6 Personen.

Ich fand also eine Person, die bei Schluss der Behandlung nur einzelne Cylinder hatte, trotzdem ihr 30 Ueberstreichungen gegeben worden waren; bei allen den übrigen $\mathrm{Pa}$ tienten fand ich dagegen eine Vermehrung der Cylinder, die bei 6 sogar höchst bedeutend war. Bei 2 Patienten war die Vermehrung der Cylinder nur unbedeutend, aber so bekam der eine auch nur 20 Ueberstreichungen, und bei dem anderen trat nach einer Einspritzung von Thymolquecksilber und 5 Ueberstreichungen Stomatitis auf, so dass die Behandlung abgebrochen werden musste, Bei keiner dieser beiden Personen waren bei Beginn der Behandlung Cylinder zu entdecken gewesen, aber 
trotz der geringen Behandlung mit Hg stellten sich nicht nur Cylinder ein, sondern sie vermehrten sich auch ein wenig, und es ist sehr wahrscheinlich, dass wir auch hier eine bedeutende Menge Cylinder bekommen haben würden, wenn die Behandlung länger gedauert hätte. Alle diese Fälle zeigen jedoch, dass man auch da, wo sich eine gelinde Albuminurie findet, eine kräftige Hg-Behandlung ohne ersichtlichen Schaden für den Patienten geben $\mathrm{kann}$.

Dieses kann ja für eine so unbedeutende Albuminurie wie die in dem obenerwähnten Falle gelten, aber wis ist das Verhältniss dann, wenn sich bei Beginn der Behandlung ein schwereres Nierenleiden findet? Ich stellte diese Frage auch in meinem vorigen Aufsatz, und ich will nun einige Worte daraus anführen: "Wenn man nun sieht, welche Reizung die Hg-Elimination auf eine gesunde Niere ausüben kann, hat man daun das Recht, die $\mathrm{Hg}$-Behandlung oder, richtiger, eine kräftige Hg-Behandlung bei einem Patienten anzuwenden, welcher z. B. eine chronische Nephritis hat? Es ist hervorgehoben worden, dass eine nicht gesunde Niere das Quecksilber nicht in hinreichender Menge eliminiren könne, welches dann für seine Elimination andere Wege, speciell die Speicheldrüse suche und dadurch Anlass zu einer schweren Stomatitis geben könne. Diese Auffassung ist sicher in vielen Hinsichten nicht richtig. Es wird, selbst bei der schwersten Stomatitis, nur eine höchst unbedeutende Menge $\mathrm{Hg}$ durch den Speichel eliminirt; dieses hat Schmidt gezeigt, und auch meine eigenen, voraus publicirten Untersuchungen haben es ergeben. Kann eine kranke Niere das $\mathrm{Hg}$ nicht in hinreichender Menge eliminiren, so wird das übrige $\mathrm{Hg}$ ganz sicher auf dem Wege eliminirt, auf dem seine Elimination normaliter in grosser Menge stattfindet, $\mathrm{d} . \mathrm{h}$. durch die Foeces. Sowohl Schuster, wie ich haben nachgewiesen, dass bei der Einreibungscur $\mathrm{Hg}$ in bedeutender Menge durch die Foeces abgeht; ich habe gezeigt, dass dieses auch bei verschiedener Injectionsbehandlung der Fall ist. Uebrigens kann eine kranke Niere (ehronische Nephritis) sehr gut eine grosse Menge $\mathrm{Hg}$ eliminiren; so fanden sich z. B. in $350 \mathrm{Gr}$. Urin, 1.012 spec. Gewicht, von dem Fall 38 den 6./5. nach 30 Benzoe-Hg-Einspritzungen eine grosse Menge recht ansehnliche Hg-Kügelchen ; 
in 360 Gr. Urin, 1.020 spec. Gewieht, von dem Fall 33 den 21./4. nach 7 Thymol-Hg-Linspritzungen einige kolossale und viele kleine $\mathrm{Hg}$-Kügelchen;

in $320 \mathrm{Gr}$. Urin, 1,019 spec. Gewicht, von dem Fall 41 den 27./6. nach 10 Einreibungen eine bedeutende Menge, doch nur kleinere Hg-Kügelchen.

Auch durch eine kranke Niere kann somit eine bedeutende Menge $\mathrm{Hg}$ ausgeschieden werden. Das Wichtigste ist natürlicherweise aber: kann dieses ohne Schaden für die Nieren geschehen? Dieses ist eine Frage, welche wir jetzt noch nicht beantworten können, doch kann ich einen Fall anführen, in welchem die Nieren durch die Hg-Elimination keinen sichtbaren Schaden gelitten haben.

X. hatte seit vielen Monaten Nephritis gehabt, als sie sich auch Syphilis zuzog. Den 9./3. 1891 hatte sie Roseola und Papeln; bekam Th-Hg: den 9./3., 15./3., 21./3., 27./3., 2./4. und 8./4. und war symptomfrei nach der dritten Einspritzung. Ihr Albumingehalt war folgender: den 9./3. 0,13, 15./3. $0,15,21 . / 3.0,07,27 . / 3.0,70,30 . / 3.0,25, \quad 2 . / 4$. 0.07., 5./4. $0,07,8 . / 4$. $0,07,14 . / 4.0 .02,19 . / 4.0,05,24 . / 4.0,02,28 . / 4.0,04,7 . / 5.0,10,15 . / 5$. $0,10,24 . / 5$. 0,25, 2./6. 0,06, 10./6. 0,02, 18./6. 0,04 Proc. In diesem Falle konnte also eine grosse Menge Hg ohne nachweisbaren Schaden für die Nieren gegeben werden."

Seitdem habe ich Gelegenheit gehabt, 4 solche Fälle zu untersuchen.

Fall XXI. L., bekam zwischen dem 5./6. und 9./7. sieben Einspritzungen von Thymolquecksilber. Seine Albuminmenge verhielt sich während dieser Zeit in folgender Weise: den 7./6. 0,03, 15./6. 0.03, 20./6. 0,03, 26./6. 0,07. 2./7. 0,05, 9./7. 0,05, 16./7. 0,07 Procent. Das Procent stieg zwar, doch nicht in einem solchen Grade, dass ich es für nöthig erachtete, die Behandlung abzubrechen.

Fall XXII M., hatte chronische Nephritis, ehe er sich Syphilis zuzog, die er nun seit mehreren Jahren gehabt hatte. Er hatte im April Gummata und wurde dafür mit sublimateinspritzungen behandelt. Der Albumingehalt war den 20./4. 1,0, 26./4. 1,03, 8./5. 0,8, 13./5. 0,7, 18./5. 0,55, 25./5. 0,65 Procent. In diesem Falle sank der Albumingehalt trotz der $\mathrm{Hg}$ Behandlung, und der Patient vertrug die $\mathrm{Hg}$-Elimination durch die Nieren sehr gut.

In dem folgenden Falle hinwieder stieg der Albumingehalt, trotzdem ich seinetwegen die Einspritzungen von Thymolquecksilber nur mit längeren Zwischenräumen gab.

Fall XXIII. G., bekam Th-Hg-Einspritzungen den 12./6., 27./6., 7./7. und 16./7. Der Albumingehalt war den 8./6. 0,04, 17./6. 0,03, 25./6. 0,15 
8./7. 0,07 und 19.77. 0,4 Procent. Da die Albuminurie sich vermehrte, so fand ich es räthlich, mit der Hg-Behandlung aufzuhören. Der Patient war bereits frei von Symptomen.

Ein gleiches war das Verhältniss in folgendem Fall, wo leider das Procent des Albumins nicht bestimmt wurde, wo aber doch eine für das blosse Auge sichtbare, bedeutende Vermehrung des Eiweisses entstand, während gleichzeitig sowohl der Cylindergehalt, wie dessen Beschaffenheit eine Veränderung erlitt.

Fall XXIV. F., bekam zwischen dem 7./8. und 22./8. Ueberstreichungen.

Den 7./8. Alb.; 1,012 spec. Gew.; viel Cylinder.

Den 12./8. " $1,010 "$ " " "

Den 17.8. Alb. vermehrt; 1,012 spec. Gew.; viele und grosse Cylinder mit Zellen.

Den 22./8. Alb. noch mehr vermehrt; 1,014 spec. Gew.; eine höchst bedeutende Menge Cylinder und Zellen.

Ich wagte es mun nicht, mit der Behandlung länger fortzufahren, besonders da die Patientin jetzt frei von Symptomen war. Der Harn wurde am 27./8. und 29./8. untersucht, wobei es sich zeigte, dass der Albumingehalt fortfahrend zunahm; beide Male fanden sich eine höchst bedeutende Menge Cylinder, von denen eine grosse Menge Zellen hatte.

Diese Fälle zeigen, wie während einer Hg-Behandlung bei einer chronischen Nephritis sowohl die Albuminmenge, wie der Cylindergehalt sehr zunehmen kann. Sie berechtigen mich, dieselbe Ansicht wie in meinem vorigen Aufsatz auszusprechen, nämlich dass man Patienten mit krankhaft veränderten Nieren nur mit Vorsicht und unter beständiger Controlirung der Beschaffenheit des Harnes mit Quecksilber behandeln darf wenigstens wenn es sich um eine kräftige solche Behandlung handelt.

Sollte ich die Ergebnisse dieser meiner Untersuchungen zusammenfassen wollen, so würde ich hier dieselben Schlüsse wie in meinem vorigen Aufsatz ziehen können, die ich bereits im Anfange dieses Aufsatzes citirt habe. Diese meine letzten Untersuchungen haben in allem die Richtigkeit der Ansichten über die $\mathrm{Hg}$-Albuminurie und $\mathrm{Hg}$-Cylindrurie bestätigt, die ich damals glaubte aussprechen zu dürfen. 